\title{
INFLUENCIA DE TRES FITOHORMONAS PARA LA PROPAGACIÓN VEGETATIVA SOBRE DOS TIPOS DE ESQUEJES DE KA' A HE' E (Stevia rebaudiana), CON TRES DOSIS DE POTASIO Y SU EFECTO SOBRE EL RENDIMIENTO DEL CULTIVO EN LA ZONA DE SANTO DOMINGO DE LOS COLORADOS
}

\author{
Autores: \\ Luis Gusqui' \\ César Huisha' \\ Miguel Romero' \\ Edison Bermúdez ${ }^{1}$
}





\section{Resumen}

Esta investigación está encaminada a evaluar el efecto de fitohormonas sobre la propagación vegetativa de esquejes y su comportamiento en el campo de acuerdo a la fertilización de potasio en el cultivo de Stevia (Stevia rebaudiana). La investigación se efectuó en la Granja Experimental de la Carrera de Ingeniería Agropecuaria de la Universidad Tecnológica Equinoccial, Campus Santo Domingo. Para la propagación se usaron 8000 esquejes tanto apicales como sub apicales, en bandejas plásticas germinadoras de 128 hoyos con substrato para enraizamiento. En el campo experimental se trasplantó 7000 plantines con un distanciamiento en doble hilera de $70 \mathrm{~cm} \times 30 \mathrm{~cm} \times 20$ cm. Los tratamientos evaluados fueron: tres fitohormonas sobre dos tipos de esqueje apical y sub apical para el enraizamiento y tres dosis de Potasio sobre el rendimiento del cultivo. Para la primera etapa se utilizó un Diseño de Bloques Completos al Azar con arreglo Bifactorial (3x2), con cuatro repeticiones. La siguiente etapa se manejó un Diseño de Bloques Completos al Azar con Arreglo Bifactorial (2x3+2) con tres repeticiones, para el análisis de medias en los dos casos se realizó mediante la prueba de Tukey al $5 \%$ de igual forma comparaciones ortogonales de los tratamientos en estudio. Las variables evaluadas para el enraizamiento de esquejes fueron: \% de prendimiento de esquejes, días al trasplante, número y longitud de raíces, para el efecto del potasio en el campo experimental fueron: Altura de planta, diámetro del tallo, regeneración vegetativa, rendimiento, factor parcial de productividad, eficiencia agronómica, eficiencia de recuperación, eficiencia de remoción, eficiencia fisiológica, concentración de nutrientes en la hoja y monitoreo de plagas y enfermedades. En el enraizamiento los esquejes tipo apical reportan el mayor porcentaje rizogénico en menor tiempo con un porcentaje de prendimiento de 97,72 \%; con la acción de la concentración de auxinas sintéticas o ácidos orgánicos a base de Hormonagro, siendo un regulador fisiológico para las plantas, afectando los puntos de crecimiento en diferentes procesos y promoviendo la emisión radical en los diferentes tipos de esquejes. La dosis de potasio evaluados en el rendimiento productivo de la Stevia, no reporto significancia estadística en la acumulación y formación de materia seca. La influencia del potasio se traduce en mejorar la calidad de la planta, promoviendo el desarrollo de tejidos y el mantenimiento adecuado del agua en la planta, siendo un importante factor para el funcionamiento de los procesos fisiológicos.

Palabras clave: Stevia, fitohormonas, propagación vegetativa, rendimiento 


\section{Abstract}

This research aims to evaluate the effect of phytohormones on vegetative propagation of cuttings and their behavior in the field, to potassium fertilization in the cultivation of Stevia (Stevia rebaudiana). The research was conducted at the Experimental Farm of Agricultural Engineering Career Equator Technological University, in Santo Domingo. For propagating cuttings 8000 were used as sub apical apical both in plastic trays with holes 128 germinating substrate for rooting. In the experimental field was transplanted 7000 seedlings with a double row spacing of $70 \mathrm{~cm} \times 30 \mathrm{~cm} \times 20 \mathrm{~cm}$. The treatments were: three phytohormones on two types of apical and sub apical cuttings for rooting and three doses of potassium on crop yield. For the first stage we used a block design under Random Bifactorial ( $3 \times 2$ ), with four replications. The next stage managed a block design under Random Bifactorial $(2 \times 3+2)$ with three replicates for analysis of means in the two cases was performed using the Tukey test at 5\% Similarly the orthogonal comparisons study treatments. Variables evaluated for rooting cuttings were:\% of surviving cuttings, transplant days, number and length of roots, to the effect of potassium in the experimental field were: plant height, stem diameter, vegetative regeneration, performance, partial factor productivity, agronomic efficiency, recovery efficiency, removal efficiency, physiological efficiency, nutrient concentrations in leaf and pest and disease monitoring. Rooting the cuttings in the apical type rhizogenic report the highest percentage in a shorter time with a percentage of seizure of $97.72 \%$, with the action of the synthetic auxin concentration or organic acids Hormonagro base, being a physiological regulator for plants, affecting growth points in different processes and promoting radical emission in different types of cuttings. The potassium doses evaluated in the yield of Stevia, reported no statistical significance in the formation and accumulation of dry matter. The influence of potassium results in improving the quality of the plant tissues to promote the development and maintenance of adequate water in the plant, being an important factor for the performance of physiological processes.

Keywords: Stevia, phytohormones, vegetative propagation, performance 


\section{Introducción}

La Stevia puede ser propagada por semillas, pero no se logra un buen porcentaje de germinación. Además, por ser una especie alógama (alta tasa de cruzamiento) existe un alto porcentaje de intercambio genético (alta variabilidad en los cultivos) y la población obtenida a partir de semillas, puede presentar variaciones por fenómenos de segregación genética. (Ballevé, 1992).

Dada la variabilidad genética se puede ocasionar un cultivo con plantas de características muy disímiles entre sí; entonces lo conveniente es la clonación, la reproducción asexual (“de gajo”), a partir de plantas de características deseadas. La calidad y cantidad de brotes que conforman cada cepa están directamente relacionadas, entre otros factores, con la edad y el manejo del cultivo que se ha de utilizar para la obtención del material de propagación. Otra forma de propagación vegetativa es a través de estacas; método que convenientemente ajustado podría ser usado a escala comercial.

La agricultura moderna utiliza la manipulación de los cultivos mediante la aplicación de fitohormonas para estimular el crecimiento de las plantas, con la finalidad de obtener altos rendimientos. Esta nueva tendencia encaminada a usar materiales orgánicos que proporcionen los requerimientos necesarios para el desarrollo de cada especie cultivada. Hay varios abonos o fertilizantes que son permitidos en agricultura orgánica, de los que se debe tener toda la información posible y su composición química, (Enríquez, 2003).

La propagación por esquejes, por división de coronas o por micropropagación (cultivos de tejidos) son procesos vegetativos que garantizan el mismo genotipo, pero a mayores costos. Tales procesos, con excepción al de cultivos de tejidos, presentan la limitante en el número de plantas que se obtienen en función de la planta madre. Por lo tanto la propagación más indicada es aquella por división de la corona y requiere alta cantidad de mano de obra calificada. El tiempo de formación de las plántulas en vivero es de 40-60 días. (Martínez, 2000)

La mejor época para el trasplante en zonas templadas es durante la primavera. Este periodo coincide con el de mayor desarrollo vegetativo ya que la planta no florece en los días largos y es conveniente para la concentración de Steviósido. La densidad es de 130.000 plha-1 con distancias de $0.15 \mathrm{~m}$ entre hilera y $0.5 \mathrm{~m}$ entre surco. (Martínez, et. al. 2000).

En la agricultura moderna, el suplemento natural $\mathrm{K}$ de los suelos no es adecuado para mantener rendimientos altos. Por esta razón los contenidos del suelo deben ser suplementos con fertilización de potasa, la cual incrementa la cantidad de $\mathrm{K}$ inmediatamente disponible para ser tomado por los cultivos. No se cuenta con estudios sobre el cultivo de Ka' a he' e (Stevia rebaudiana), la poca información empírica se la puede obtener es de investigaciones e introducción del cultivo que se ha realizado en otros países donde ya ha sido demostrado el potencial que puede tener así como su correcto procesamiento industrial.

Resultados de estudios realizados en Japón demostraron que los niveles máximos de materia seca acumulada de plantas de Stevia contienen 1.4\% N, 0.3\% P, y 2.4\% K (Katayama, et al. 1976). En Ontario la producción total de biomasa es de $7500 \mathrm{Kg}$ ha-1, correspondiente a $26 \%$ de raíces, $35 \%$ tallos y $39 \%$ de hojas; para obtener esta biomasa óptima se requiere aproximadamente 105 Kgha-1 N, 23 Kgha-1 P, 180 Kgha-1 K en el suelo. La dosis de fertilizante a aplicar debe calcularse teniendo en cuenta el tipo de suelo y la necesidad de optimizar cada situación específica. Se recomienda fertilización orgánica y mineral tomando como base 100 Kgha-1 de N-P-K. (Martínez, 2000).

El potasio $(K)$ es un macro nutriente esencial requerido en grandes cantidades para el normal crecimiento y desarrollo de los cultivos. Algunas de las principales funciones de las plantas donde el $\mathrm{K}$ 
Influencia de tres fitohormonas para la propagación vegetativa sobre dos tipos de esquejes de Ka' a he' e (Stevia rebaudiana), con tres dosis de potasio y su efecto sobre el rendimiento del cultivo en la zona de Santo Domingo de los Tsáchilas.

está comprometido son: la osmoregulación, la síntesis de los almidones, la activación de enzimas, la síntesis de proteínas, el movimiento estomático y el balance de cargas iónicas (Maathuis y Sanders, 1994; Marschner, 1995). Cantidades adecuadas de potasio son importantes contribuyentes en la adaptación de los cultivos al stress causado por factores bióticos y abióticos, tales como sequías, salinidad, heladas, ataques de insectos o enfermedades (Kafkafi, 1997).

\section{Materiales y métodos}

El presente ensayo se evaluó desde marzo del 2011 a enero del 2012 que comprenden las etapas de enraizamiento y desarrollo del cultivo. El experimento estuvo ubicado en la Granja Experimental de la Escuela de Ingeniería Agropecuaria en la Universidad Tecnológica Equinoccial, Campus Santo Domingo, ubicado en el Km 4⿳1⁄2 vía Chone con latitud $00^{\circ} 14^{\prime}$ S, longitud $79^{\circ} 11^{\prime} \mathrm{W}$ y altitud de $552 \mathrm{~m}$. s.n.m. Se valoró el enraizamiento de dos tipos de esquejes con el uso de fitohormonas presentes en tres productos comerciales y el rendimiento de la Stevia con la utilización de tres dosis de potasio. La descripción de los tratamientos se presenta en la tabla 1 y 2. Se utilizó para los dos casos un diseño de bloques completos alazar con arreglo bifactorial, para el primer caso con seis tratamientos y cuatro repeticiones, para el segundo caso ocho tratamientos y tres repeticiones dando un total de 24 unidades experimentales. Para la comparación de medias de los tratamientos se utilizó la prueba de Tukey al 5\% y comparaciones ortogonales de los tratamientos en estudio. Los parámetros evaluados para el primer caso fueron porcentaje de prendimiento de esquejes, días al trasplante, número y longitud de raíces. El porcentaje de prendimiento se registró a los 7 y 14 días de la siembra de los esquejes en las bandejas germinadoras, tomando como referencia para esta variable, el total de esquejes sembrados por tratamiento, días al trasplante se contabilizó desde el momento de la siembra en las bandejas hasta la siembra de los plantines en el campo experimental, número y longitud de raíces se tomó de diez plantas alazar, en la misma se contabilizó las raíces presentes por planta, para la longitud se recolectó los datos, midiéndolo desde el cuello del tallo hasta el punto terminal de la raíz más larga. Para la fase de campo los parámetros evaluados fueron: Altura de planta, diámetro del tallo, regeneración vegetativa, rendimiento, factor parcial de productividad, eficiencia agronómica, eficiencia de recuperación, eficiencia de remoción, eficiencia fisiológica, concentración de nutrientes en la hoja y monitoreo de plagas y enfermedades. La altura de planta se registró midiéndolo desde el suelo hasta el punto terminal del ápice más alto, el diámetro de tallo se midió a $5 \mathrm{~cm}$ de altura desde el suelo, la regeneración vegetativa se procedió a contabilizar el número de brotes tiernos regenerados después de cada cosecha, el rendimiento se pesó la cantidad de hoja fresca y seca por tratamiento, para el factor parcial de productividad se registró los kilogramos de hoja cosechada por kilogramo de potasio aplicado, la eficiencia agronómica se contabilizó con la relación entre el aumento en la producción por kilogramo de elemento aplicado, en la eficiencia aparente de recuperación se tomó en cuenta los kilogramos de aumento en la absorción por la cantidad de elemento aplicado en kilogramos, la eficiencia fisiológica se tomó en cuenta la relación del aumento en la producción por el aumento en la absorción del elemento en kilogramos, la concentración de nutrientes en las hojas, para este parámetro se recolectó muestras de materia verde, se identificó y se envió al laboratorio para el análisis de macro y microelementos y para la incidencia de plagas y enfermedades, se efectuaron observaciones quincenales para identificar insectos y agentes fitopatógenos que afecten al cultivo de Stevia. 


\begin{tabular}{|c|c|l|}
\hline Simbología & Código & \multicolumn{1}{|c|}{ Tratamientos } \\
\hline T1 & A1B1 & Hormonagro + esqueje apical \\
T2 & A1B2 & Hormonagro + esqueje sub apical \\
T3 & A2B1 & Rootex + esqueje apical \\
T4 & A2B2 & Rootex + esqueje sub apical \\
T5 & A3B1 & Raizal + esqueje apical \\
T6 & A3B2 & Raizal + esqueje sub apical \\
\hline
\end{tabular}

Tabla 1. Descripción de los tratamientos para el enraizamiento de esquejes, evaluados en la investigación.

\begin{tabular}{|c|c|l|}
\hline Simbología & Código & \multicolumn{1}{|c|}{ Tratamientos } \\
\hline T1 & A1K1 & Esqueje apical + Dosis baja de Potasio \\
T2 & A1K2 & Esqueje apical + Dosis media de Potasio \\
T3 & A1K3 & Esqueje apical + Dosis alta de Potasio \\
T4 & A2K1 & Esqueje sub apical + Dosis baja de Potasio \\
T5 & A2K2 & Esqueje sub apical + Dosis baja de Potasio \\
T6 & A2K3 & Esqueje sub apical + Dosis baja de Potasio \\
T7 & To & Testigo absoluto esqueje apical \\
T8 & Ti & Testigo absoluto esqueje sub apical \\
\hline
\end{tabular}

Tabla 2. Descripción de los tratamientos para el efecto de potasio en el rendimiento de Stevia.

\section{Resultados y discusión}

\section{PROPAGACIÓN VEGETATIVA}

\section{Porcentaje de prendimiento de esquejes (\%)}

En el Tabla 3 se observa los resultados del análisis de varianza para el porcentaje de prendimiento evaluado a los 15 días. Se muestra alta significancia entre las fitohormonas (factor A) y significancia en los tipos de esqueje de Stevia rebaudiana (factor B), no se observa ninguna diferencia estadística en el resto de fuentes. Por lo tanto existe influencia de las fitohormonas en el enraizamiento de esquejes, que cubre la necesidad de producción de material vegetativo, preservando sus cualidades genéticas. El coeficiente de variación fue de $7.9 \%$ indicando confiabilidad en la experimentación.

\begin{tabular}{llllll}
\hline \multicolumn{1}{c}{ F.V. } & gl & SC & CM & F & \\
\hline Total & 23 & 2464.32 & & & \\
Factor A & 2 & 913.3 & 456.65 & 8.28 & $* *$ \\
Factor B & 1 & 336.3 & 336.3 & 6.1 & $*$ \\
A*B & 2 & 235.16 & 117.58 & 2.13 & \\
Repeticiones & 3 & 152.59 & 50.86 & 0.92 & \\
Error & 15 & 826.98 & 55.13 & & \\
\hline \multicolumn{7}{l}{ Coeficiente de Variación (\%): } & 7.90 & & & \\
\hline
\end{tabular}

Tabla 3. Análisis estadístico del \% de prendimiento de esquejes

En la Figura 1 se presentan los datos promedio del efecto de las fitohormonas en el \% de prendimiento de esquejes apicales y sub apicales. El factor A3 (Raizal) con $99.42 \%$ y A1 (Hormonagro) con $97,17 \%$ en prendimiento, son estadísticamente iguales y superior al factor A2 (Rootex). Esto indica que el producto raizal y hormonagro influyen positivamente sobre la variable en mención, confirmando la hipótesis de que las fitohormonas ejercen una influencia positiva sobre la propagación vegetativa asexual de esquejes del cultivo de Stevia.

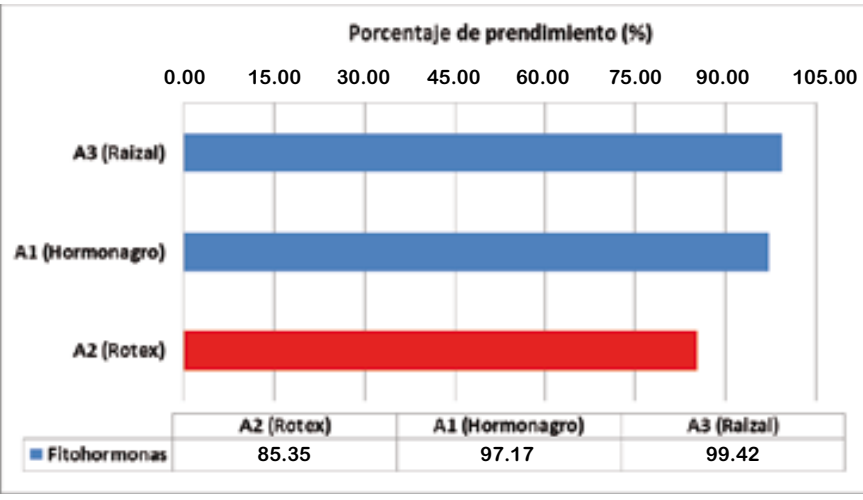

Figura 1. Eficiencia de Fitohormonas (A) en el prendimiento de esquejes (\%)

Bañón et al., 2002 cita: que la aplicación de reguladores del crecimiento puede aumentar el enraizamiento. El compuesto más comúnmente utilizado para estimular la formación de raíces adventicias de esquejes de tallo es el ácido indolbutírico (AIB) está implicado en los procesos fisiológicos de la formación de raíces en esquejes. Los resultados expuestos en el Figura 1 concuerda con lo expuesto por Bañón, 2002, quien dice que hormonagro y raizal son fitorreguladores compuestos por auxinas (AIB) en diferentes concentraciones, que permite un alto porcentaje de germinación.

111 
Influencia de tres fitohormonas para la propagación vegetativa sobre dos tipos de esquejes de Ka' a he' e (Stevia rebaudiana), con tres dosis de potasio y su efecto sobre el rendimiento del cultivo en la zona de Santo Domingo de los Tsáchilas.

En la Figura 2, se observa los resultados del Factor B (tipos de esquejes) y según Tukey al $5 \%$ de probabilidad, se tiene dos rangos de significancia. En primer lugar con el mayor promedio en prendimiento están los esquejes de tipo Apical (B1) con un $97.72 \%$ y garantiza un alto porcentaje de prendimiento y su posterior enraizamiento. A diferencia de los esquejes sub apicales o laterales (B2) que presentan un menor índice de prendimiento con 90.24\% que lo ubica en el segundo rango de significancia estadística. Esto indica que los esquejes de tipo apical tienen mayor porcentaje de enraizamiento en el cultivo de Stevia rebaudiana que corrobora con lo mencionado por Bredmose et al., 2001, quien manifiesta que la obtención de un sistema radicular de mayor peso y por tanto de mayor desarrollo, está relacionado con el peso seco del esqueje utilizado, lo que en principio podría hacer pensar de utilizar aquellos de mayor grosor y un incremento de la rizogénesis es mayor en esquejes apicales.

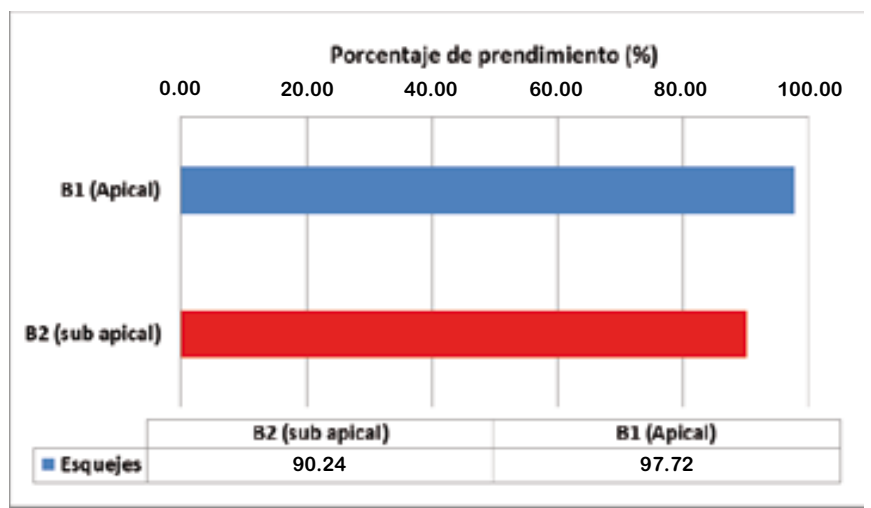

Figura 2. Porcentaje de prendimiento de tipos de esquejes (B).

\section{Longitud de raíces (cm.) de esquejes de Stevia rebaudiana}

Los datos del análisis de varianza de la longitud de raíces se presentan en el Tabla 4, donde no reportan diferencias significativas a los 21 días de evaluación, confirmando la hipótesis que las fitohormonas no ejercen una influencia positiva sobre la propagación vegetativa asexual de los esquejes de Stevia. El coeficiente de variación fue de $17.37 \%$.

\begin{tabular}{lllll}
\hline \multicolumn{1}{c}{ F.V. } & gl & SC & CM & F \\
\hline Total & 23 & 31.44 & & \\
Factor A & 2 & 0.74 & 0.37 & 0.21 \\
Factor B & 1 & 1.17 & 1.17 & 0.66 \\
A*B & 2 & 0.33 & 0.16 & 0.09 \\
Repeticiones & 3 & 2.66 & 0.89 & 0.5 \\
Error & 15 & 26.54 & 1.77 & \\
\hline Coeficiente de Variación (\%): & 17.37 & & \\
\hline
\end{tabular}

Tabla 4. Análisis de varianza de la longitud de raíces de esquejes.

Al momento de hacer enraizar una estaca, son varios los factores que inciden en este proceso, pero para su mejor análisis y comprensión se dividirán en tres grandes grupos: el primero de ellos corresponde a las características relacionadas con el material vegetal a propagar; en segundo lugar están los tratamientos aplicados a las estacas y, por último se encuentran las condiciones ambientales a que son sometidas las estacas durante el enraizamiento (Cabello, 2000).

\section{Número de raíces por esqueje de Stevia rebaudiana}

En la Tabla 5, se presentan los resultados del análisis estadístico del número de raíces, obtenidos en el enraizamiento de los esquejes. Se observa que existen diferencias estadísticas altamente significativas entre las fitohormonas (factor A) y ninguna significancia en el resto de fuentes en estudio. La influencia de la aplicación de reguladores de crecimiento aumentan la proliferación de raíces en los esquejes de Stevia rebaudiana. El valor del coeficiente de variación fue de $23.10 \%$, aceptables para este parámetro de evaluación.

\begin{tabular}{llllll}
\hline \multicolumn{1}{c}{ F.V. } & $\mathrm{gl}$ & $\mathrm{SC}$ & $\mathrm{CM}$ & $\mathrm{F}$ \\
\hline Total & 23 & 957.49 & & & \\
Factor A & 2 & 696.91 & 348.46 & 25.98 & $* *$ \\
Factor B & 1 & 17.51 & 17.51 & 1.31 & \\
A*B & 2 & 26.16 & 13.08 & 0.98 \\
Repeticiones & 3 & 15.72 & 5.24 & 0.39 \\
Error & 15 & 201.19 & 13.41 & & \\
\hline \multicolumn{6}{l}{ Coeficiente de Variación (\%): } \\
\hline
\end{tabular}

Tabla 5. Análisis de varianza para el número de raíces de esquejes de Stevia rebaudiana. 
En la Figura 3 se muestran los datos del número de raíces por esqueje apical y sub apical. El factor A1 (Hormonagro) con una proliferación de 23.47 raíces/esqueje, es superior y diferente al factor A2 (Rootex) y A3 (Raizal) con 12.31 y 11.78 raíces/esqueje respectivamente. Según Vivanco, 2009, el comportamiento de Hormonagro, destaca como estimulante para el enraizamiento de los esquejes, actuando mejor debido a las auxinas presentes en ellos, las mismas que ejercen un efecto positivo en la formación del callo de los esquejes y el crecimiento en espesor de los tallos, provocando la división de las células del cambium que estimulan fuertemente la rizogénesis de los esquejes. Los valores alcanzados en este ensayo concuerdan con Vivanco, obteniendo el tratamiento a base de hormonagro, la mayor incidencia de raíces por esqueje que el resto de reguladores de crecimiento evaluados a los 21 días.

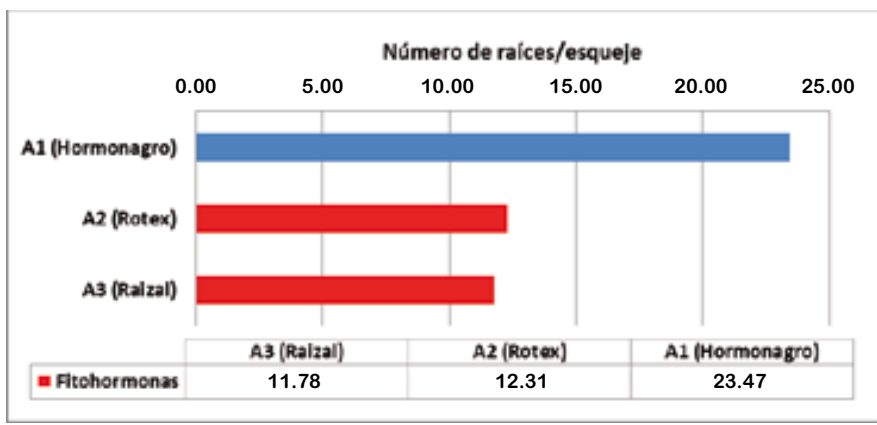

Figura 3. Eficiencia de Fitohormonas (A) en la proliferación de raíces de esqueje de Stevia rebaudiana.

\section{Días al trasplante de esquejes de Stevia rebaudiana}

De acuerdo con el análisis de varianza Tabla 6, presentó alta significancia estadística en el factor A (fitohormonas) y ninguna diferencia significativa en el resto de fuentes. Esto indica que los productos a base de fitohormonas influyen positivamente en los días al trasplante. El coeficiente de variación se mantuvo en $10.13 \%$; que nos indica confiabilidad en la experimentación.

\begin{tabular}{llllll}
\hline \multicolumn{1}{c}{ F.V. } & gl & SC & CM & F \\
\hline Total & 23 & 624.66 & & & \\
Factor a & 2 & 454.67 & 227.33 & 25.98 & $* *$ \\
Factor b & 1 & 11.41 & 11.41 & 1.30 & \\
Factor a*Factor b & 2 & 17.07 & 8.53 & 0.98 & \\
repeticiones & 3 & 10.24 & 3.41 & 0.39 & \\
Error & 15 & 131.27 & 8.75 & & \\
\hline Coeficiente de Variación (\%): & 10.13 & & \\
\hline
\end{tabular}

Tabla 6. Análisis de varianza para días al trasplante de esquejes de Stevia rebaudiana.

En la Figura 4 se presentan los datos promedios de los días al trasplante de esquejes. Se observa el efecto positivo de los productos a base de fitohormonas en esta etapa. Los tratamientos a base de Hormonagro (A1) presentaron el menor tiempo transcurrido al trasplante de esquejes de Stevia rebaudiana con 23.05 días, siendo superior y diferente al factor $\mathrm{A}_{3}$ (Raizal) con 32.49 días al trasplante y A3 (Rootex) con una media de 32.06 días, siendo estadísticamente iguales, confirmando la hipótesis de que las fitohormonas ejercen una influencia positiva sobre la propagación vegetativa asexual de esquejes de la Stevia rebaudiana. Martínez, 2000 manifiesta que la propagación por esquejes, es un proceso vegetativo que garantizan el mismo genotipo y que para asegurar el enraizamiento de los esquejes una de las condiciones fundamentales es la de conseguir que los mismos se hallen en un ambiente de alta humedad, de manera que los mismos no se deshidraten posterior al corte y que el tiempo de formación de las plántulas en vivero es de 40-60 días.

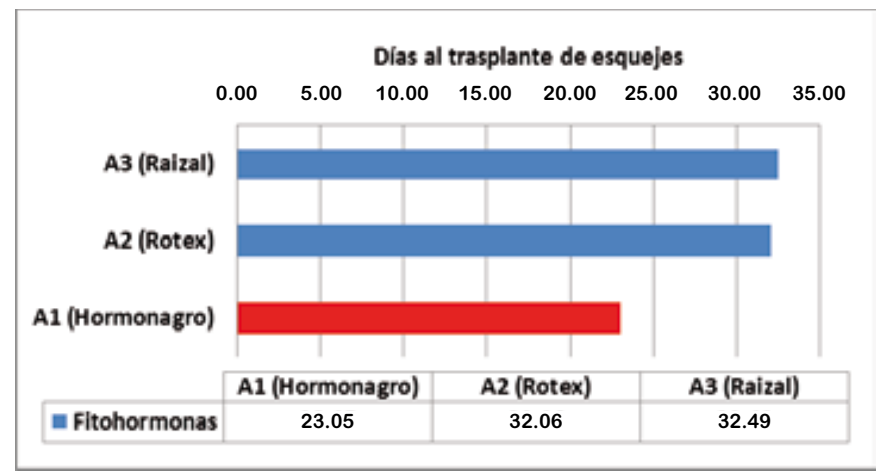

Figura 4. Eficiencia de Fitohormonas (A) en el tiempo (días) al trasplante de esquejes de Stevia rebaudiana. 
Influencia de tres fitohormonas para la propagación vegetativa sobre dos tipos de esquejes de Ka' a he' e (Stevia rebaudiana), con tres dosis de potasio y su efecto sobre el rendimiento del cultivo en la zona de Santo Domingo de los Tsáchilas.

En la Figura 5, se observa la relación entre el número de raíces/ esquejes y los días al trasplante, evaluados en un periodo de 21 días. Es determinante la proliferación radical de los esquejes que inciden en futuros platines de alta calidad al trasplante y se acorta el tiempo de vivero. En el ensayo el factor A1 (Hormonagro) se manifiesta con una proyección lineal en el desarrollo de raíces/esqueje de Stevia rebaudiana, que acorta el periodo de trasplante a partir de los 8 días con 8.94 raíces/esqueje, a los 15 días una cantidad de 16.76 raíces/ esqueje y a los 21 días un promedio de 23.47 raíces/esqueje. En menor grado en desarrollo del sistema radical los factores A3 (Raizal) y A2 (Rootex) se alarga su periodo al trasplante, y mantiene un comportamiento similar en crecimiento y números de raíces/esqueje durante el desarrollo de este ensayo, con valores que promedia a los 8 días con 4.49 y 4.69 raíces/esqueje, a los 15 días en un rango de 8.42 a 8.79 raíces/esqueje y a los 21 días la multiplicación radical es lenta con una proliferación que va de 11.78 a $12.31 \%$ de raíces/esqueje.

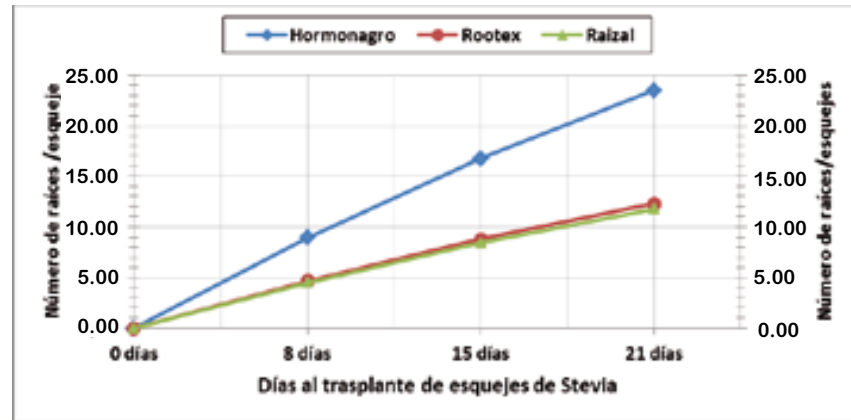

Figura 5. Relación número de raíces - Días al trasplante de esquejes de Stevia rebaudiana

\section{EN EL CAMPO DEFINITIVO}

\section{Altura de planta de Stevia rebaudiana}

En la Tabla 7 se representan los resultados del análisis estadístico de la variable altura de planta evaluados a los 15 y 30 días antes de cada corte productivo de la planta, durante nueve meses; no se observaron diferencias estadísticas significativas en las fuentes de variación en estudio de este ensayo. Las dosis de potasio no muestran efectos perceptibles sobre esta variable en el ensayo. Los valores de los coeficientes de variación fueron: 6.91, 5.63, 9.10, 7.57, 8.04 y 5.14 \%, que son aceptables.

\begin{tabular}{|c|c|c|c|c|c|c|c|c|}
\hline \multirow[b]{2}{*}{ F.V. } & \multirow[b]{2}{*}{$\mathrm{gl}$} & \multicolumn{2}{|c|}{ Primer corte } & \multicolumn{2}{|c|}{ Segundo corte } & \multicolumn{3}{|c|}{ Tercer corte } \\
\hline & & $\begin{array}{l}\text { 15día } \\
\mathrm{s}\end{array}$ & $\begin{array}{l}\text { 30día } \\
\mathrm{s}\end{array}$ & $\begin{array}{l}\text { 15día } \\
\mathrm{s}\end{array}$ & $\begin{array}{l}\text { 30día } \\
\mathrm{s}\end{array}$ & $\begin{array}{l}\text { 15día } \\
\mathrm{s}\end{array}$ & 30días & \\
\hline Total & 23 & & & & & & & \\
\hline Tratamientos & 7 & 1.48 & 1.02 & 0.22 & 0.54 & 0.53 & 2.43 & \\
\hline Factor A & 1 & 1.16 & 0.004 & 0.15 & 2.01 & 0.25 & 0.15 & \\
\hline Factor K & 2 & 0.42 & 0.31 & 0.08 & 0.25 & 0.90 & 3.39 & \\
\hline $\mathrm{A}^{*} \mathrm{~K}$ & 2 & 2.36 & 1.43 & 0.03 & 0.07 & 0.11 & 1.19 & \\
\hline Factores vs Adicionales & 1 & 1.23 & 1.01 & 0.00001 & 1.08 & 1.10 & 5.11 & * \\
\hline Testigo $_{1}$ vs Testigo ${ }_{2}$ & 1 & 2.38 & 2.62 & 1.20 & 0.08 & 0.32 & 2.61 & \\
\hline Repeticiones & 2 & 0.06 & 1.20 & 6.46 & 2.44 & 5.07 & 12.54 & \\
\hline Error & 14 & & & & & & & \\
\hline Coeficiente de Variación & ): & 6.91 & 5.63 & 9.10 & 7.57 & 8.04 & 5.14 & \\
\hline
\end{tabular}

$\begin{array}{lllllll}\text { Coeficiente de Variación (\%): } & 6.91 & 5.63 & 9.10 & 7.57 & 8.04 & 5.14\end{array}$

Tabla 7. Análisis de varianza para altura de planta de Stevia rebaudiana, durante tres cortes productivos.

De acuerdo a estos resultados se observa que en el tercer corte, existe significancia en la comparación de los factores vs adicionales. La variable altura de planta de Stevia rebaudiana, no presenta diferencias estadísticas entre los diferentes tratamientos, el crecimiento de la planta se mantiene constante en las diferentes ciclos de crecimiento. Birchler, 1998, concluye que la altura inicial de las plantas no se correlaciona, o lo hace de forma negativa, con la supervivencia, aunque sí se correlaciona con el crecimiento en altura tras la plantación, se han mostrado que la ventaja inicial en el tamaño de la planta permanece en el tiempo. Una planta ideal presenta una altura dentro de un rango que ha sido unido con el éxito en las plantaciones de una especie dada.

\section{Diámetro de tallo $(\mathrm{cm}$.}

Los datos del análisis de la varianza del diámetro del tallo se presentan en la Tabla 8. Se observa que existe alta significancia estadística en los tipos de esquejes (factor $A$ ) en el primer corte productivo de Stevia rebaudiana. En el segundo corte productivo en el diámetro de tallo, presento significancia en el factor A, mientras que en el tercer corte no presentó ninguna significancia estadística en las fuentes de estudio. Los coeficientes de variación para esta variable fueron: 5.80, 5.66 y $5.71 \%$, para las observaciones a los 15 y 30 días antes de corte productivo de la planta respectivamente. 


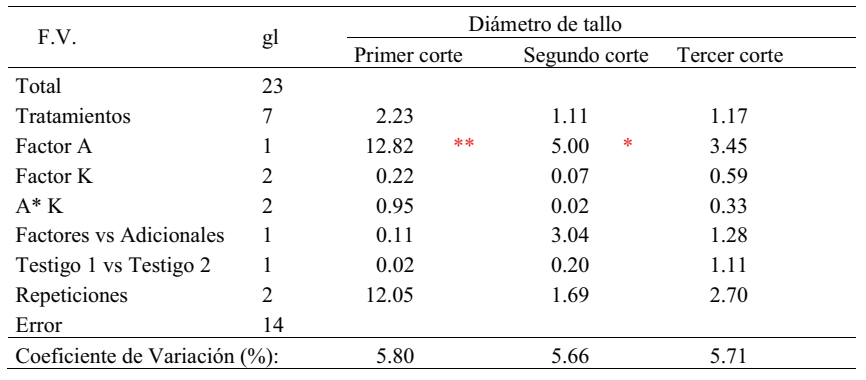

Tabla 8. Análisis de varianza para diámetro de tallo en Stevia rebaudiana, durante tres cortes productivos.

En la Figura 6 se muestra los datos promedios del efecto del Potasio en el diámetro del tallo. En el primer corte productivo marco diferencias en los tipos de esqueje (factor A) que conforman las plantas de los tratamientos que se evaluaron. Según la prueba de Tukey al $5 \%$ de probabilidad, indica que los esquejes de tipo sub apical (A2) son estadísticamente superiores con un promedio de $0.51 \mathrm{~cm}$ de diámetro, a los esquejes de propagación apical (A1) con una media de $0,46 \mathrm{~cm}$ en grosor de tallo de planta. Esto indica que el $\mathrm{K}$ influye positivamente sobre el diámetro del tallo del esqueje sub apical, confirmando la hipótesis de que una fertilización balanceada mejora las características agronómicas del cultivo de Stevia.

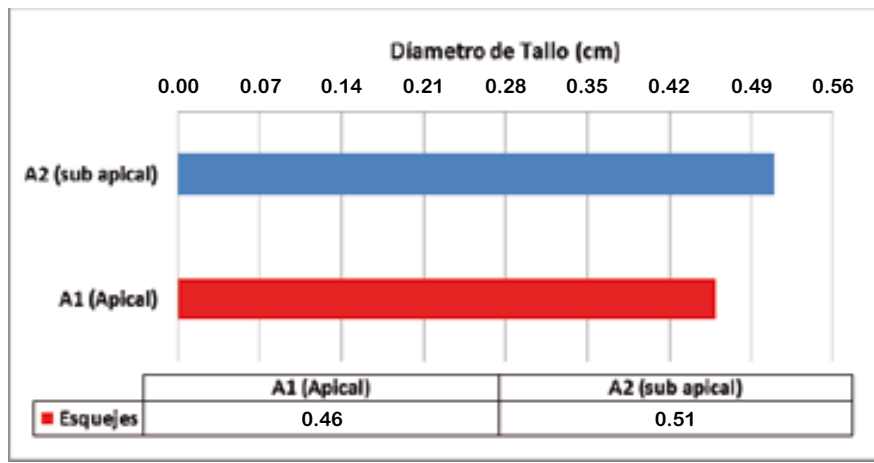

Figura 6. Diámetro de tallo de los dos tipos de esquejes en el primer corte productivo de Stevia rebaudiana.

En la Figura 7 se presenta los datos del efecto del Potasio en el diámetro del tallo. En el segundo corte productivo de la Stevia, según la prueba de Tukey al 5\% de probabilidad, presenta dos rangos significativos, el esqueje de tipo sub apical (A2) se mantiene en el primer rango con un promedio de $0.83 \mathrm{~cm}$ de diámetro, siendo superior al esqueje de propagación apical (A1) con una media de 0.79 cm en grosor de tallo de planta. Según Birchler et al 1998, indica que el diámetro del tallo da una aproximación de la sección transversal de transporte de agua, resistencia mecánica y de la capacidad relativa para tolerar altas temperaturas en la superficie del suelo. El diámetro está influenciado por la densidad del cultivo y puede verse afectado por prácticas culturales. Una planta ideal tiene un diámetro mayor que un mínimo dado.

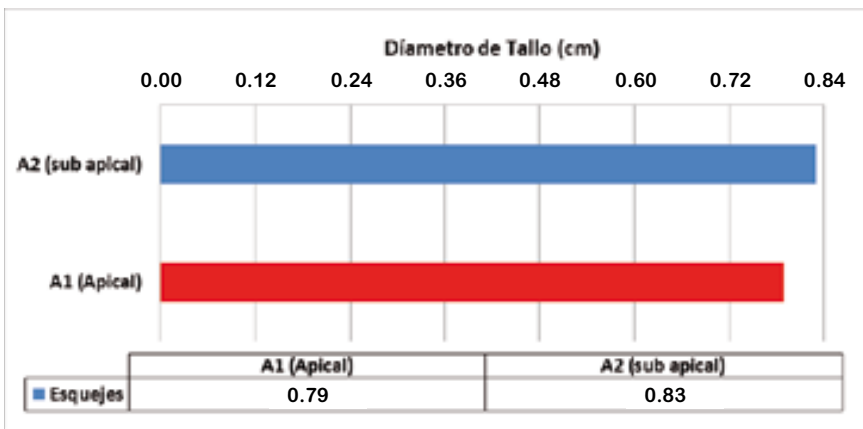

Figura 7. Diámetro de tallo de los dos tipos de esqueje en el segundo corte productivo de Stevia rebaudiana.

\section{Recuperación vegetativa de Stevia rebaudiana.}

En la Tabla 9, se reporta el resultado del análisis de varianza, durante los tres cortes productivos de Stevia rebaudiana que se evaluaron en este ensayo. Se observa alta significancia estadística entre tratamientos y tipos de esquejes (factor $A$ ), en la primera cosecha se observa la influencia significativa de las diferentes dosis de potasio (factor K) en la recuperación vegetativa de la planta. Los coeficientes de variación en esta variable fueron: 9.86, 12.61 y 14.82\% respectivamente. 


\begin{tabular}{|c|c|c|c|c|c|c|c|}
\hline \multirow{3}{*}{$\frac{\text { F.V. }}{\text { Total }}$} & \multirow{3}{*}{$\begin{array}{l}\mathrm{gl} \\
23\end{array}$} & \multicolumn{6}{|c|}{ Recuperación vegetativa } \\
\hline & & \multicolumn{2}{|l|}{ Primer corte } & \multicolumn{2}{|c|}{ Segundo corte } & \multicolumn{2}{|c|}{ Tercer corte } \\
\hline & & & & & & & \\
\hline Tratamientos & 7 & 3.68 & ** & 4.82 & ** & 4.99 & ** \\
\hline Factor A & 1 & 1.23 & ** & 16.57 & $* *$ & 14.21 & ** \\
\hline Factor K & 2 & 2.47 & $*$ & 3.65 & & 2.43 & \\
\hline$A * K$ & 2 & 2.47 & & 0.81 & & 1.16 & \\
\hline Factores vs Adicionales & 1 & 3.31 & & 7.28 & $*$ & 8.60 & $*$ \\
\hline Testigo $_{1}$ vs Testigo ${ }_{2}$ & 1 & 0.03 & & 0.95 & & 4.93 & $*$ \\
\hline Repeticiones & 2 & 10.66 & & 3.46 & & 3.42 & \\
\hline Error & 14 & & & & & & \\
\hline Coeficiente de Variación & (\%): & 9.86 & & 12.61 & & & 82 \\
\hline
\end{tabular}

Tabla 9. Análisis de varianza para la recuperación vegetativa en Stevia rebaudiana, durante tres cortes productivos.

En el segundo y tercer corte productivo se presenta significancia estadística en la comparación de los Factores en estudio (tipos de esqueje y dosis de K) vs adicionales (testigos 1 y 2 de este ensayo). De igual forma hay diferencia estadística en el tercer corte productivo entre los testigos de esta investigación. Esto muestra que el $\mathrm{K}$ influye positivamente sobre la recuperación vegetativa, confirmando la hipótesis de que una fertilización balanceada con potasio tiene efectos sobre la recuperación vegetativa de la Stevia.

Las dosis de potasio (factor $\mathrm{K}$ ) reportan influencia en la incidencia de brotes, en la primera cosecha de Stevia rebaudiana. De acuerdo con la prueba de Tukey al $5 \%$ de probabilidad, la dosis baja de potasio (K1: 120 Kgha-1) influye con la mayor proliferación de brotes con una media de 9.46 brotes/planta; mientras que la dosis media (K2: 140 Kgha-1) es estadísticamente igual con 9.28 brotes/planta y comparte un rango de significancia. La dosis alta de potasio (K3: 160 Kgha-1) es inferior con un promedio de 8.02 brotes/planta, Figura 8.

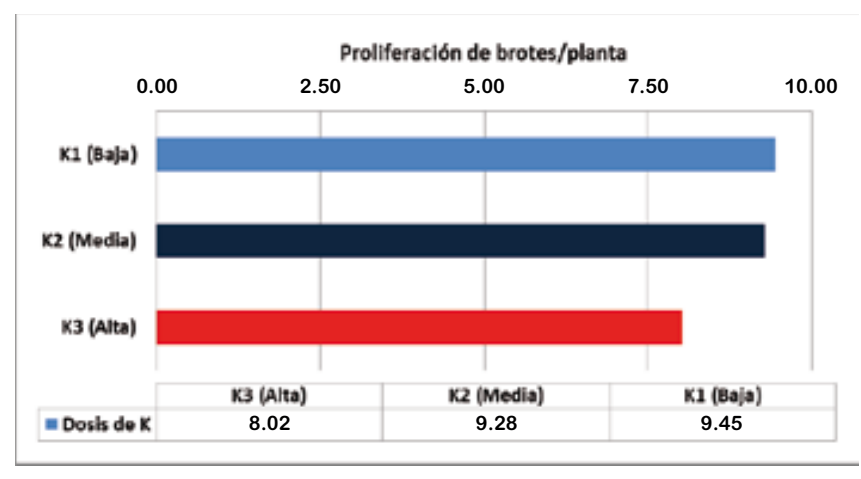

Figura 8. Incidencia del Potasio en la recuperación vegetativa de la Stevia rebaudiana en el primer corte productivo.

En la Figura 9 se observa la tendencia en recuperación vegetativa después de las diferentes cosechas productivas de hoja de Stevia rebaudiana, según el tipo de esqueje (factor $A$ ) que se uso en el trasplante. Los esquejes de tipo sub apical (A2) presentan los mejores condiciones de proliferación de brotes en las diferentes ciclos productivos, con promedios de 9.60 brotes/planta en la primera cosecha, 33.91 y 55.12 brotes/planta, en la segunda y tercera cosecha. Los esquejes apicales ( $\mathrm{A} 1$ ) con promedios inferiores de 8.23 brotes/planta durante el primer corte, 26.32 y 41.68 brotes/planta en el segundo y tercer corte respectivamente.

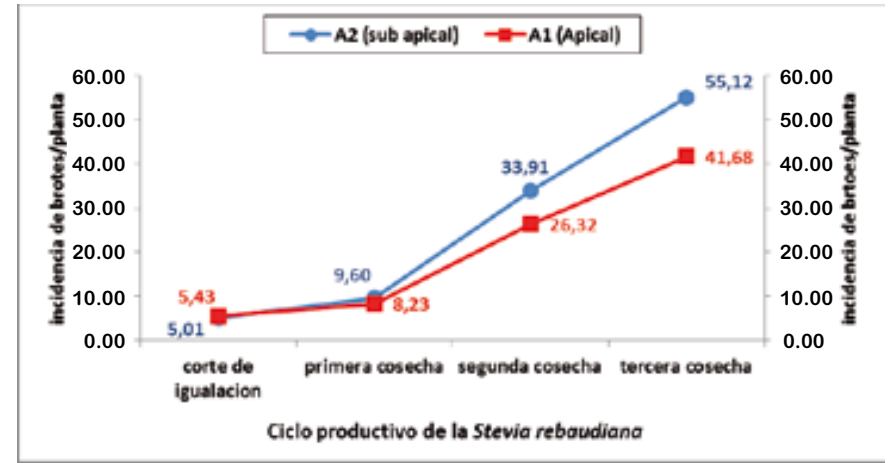

Figura 9. Comportamiento de dos tipos de esqueje, en la recuperación vegetativa de la Stevia rebaudiana durante su ciclo productivo.

La Figura 10 indica el efecto de los tratamientos de fertilización con dosis de potasio en la proliferación de brotes de la planta. El tratamiento T4 (esqueje sub apical mas dosis baja de K) presenta los

116 
mejores promedios en la primera y segunda cosecha con 10.20 y 37.97 brotes/planta, siendo superiores y diferentes al resto de tratamientos. En el último rango se encuentra los T3, con 6.93 y 23.90 brotes/planta para la primera y segunda cosecha respectivamente.

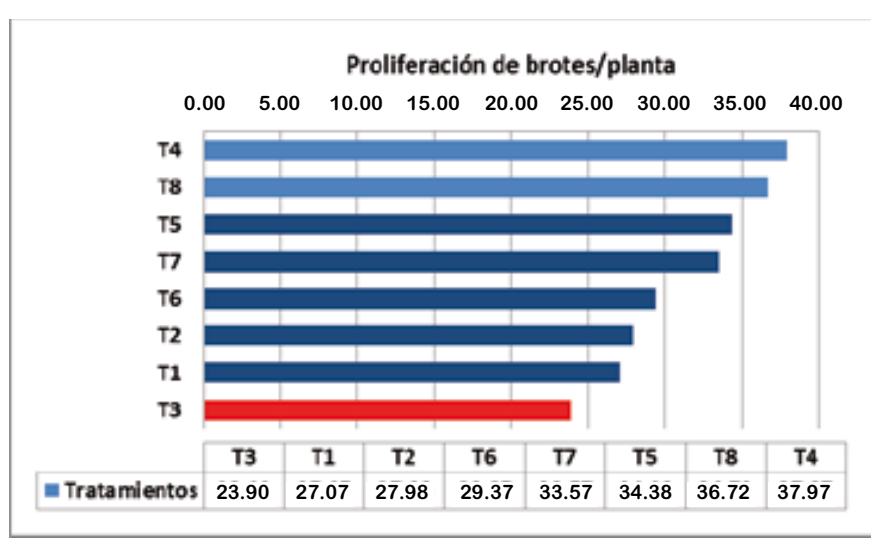

Figura 10. Proliferación de brotes en el segundo corte, en la recuperación vegetativa de la Stevia.

El tratamiento T8 (Testigo) con un promedio de recuperación vegetativa de 65.72 brotes/planta, al tercer corte (figura 11), es superior estadísticamente al resto de tratamientos. El tratamiento T1 y T3 se ubicaron en el último lugar con la más baja recuperación vegetativa de 37.35 y 37.43 brotes/planta.

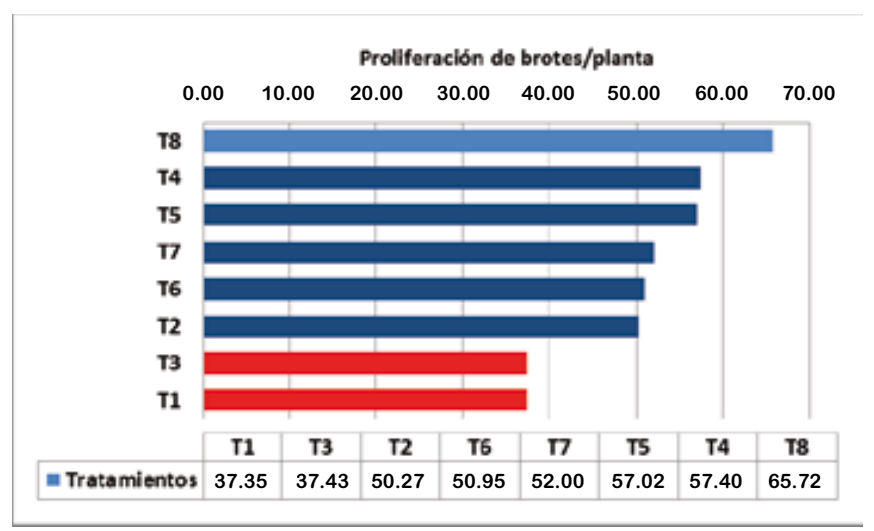

Figura 11. Proliferación de brotes al tercer corte, en la recuperación vegetativa de la Stevia.

\section{Rendimiento productivo de la Stevia rebaudiana}

En la Tabla 10 se presentan los resultados del análisis de varianza para el rendimiento productivo de hoja seca. Para los tratamientos en estudio no presentaron ninguna diferencia estadística. En el rendimiento de la hoja seca no existe incidencia significativa de las dosis de potasio evaluados en este ensayo y el incremento productivo, no difiera entre los tipos de esqueje que se usaron en el trasplante según el análisis estadístico, reportadas en las diferentes cosechas. Los coeficientes de variación que se manejaron en esta variable fueron: 5.80, 5.66 y $5.71 \%$, que nos demuestra confiabilidad en la experimentación.

\begin{tabular}{|c|c|c|c|c|}
\hline \multirow{2}{*}{ F.V. } & \multirow{2}{*}{$\mathrm{gl}$} & \multicolumn{3}{|c|}{ Rendimiento de hoja seca } \\
\hline & & Primer corte & Segundo corte & Tercer corte \\
\hline Total & 23 & & & \\
\hline Tratamientos & 7 & 0.67 & 1.20 & 0.52 \\
\hline Factor A & 1 & 2.47 & 2.76 & 0.07 \\
\hline Factor $\mathrm{K}$ & 2 & 0.36 & 0.46 & 0.66 \\
\hline $\mathrm{A} * \mathrm{~K}$ & 2 & 0.29 & 1.09 & 0.75 \\
\hline Factores vs Adicionales & 1 & 0.20 & 0.55 & 0.45 \\
\hline Testigo 1 vs Testigo 2 & 1 & 0.71 & 1.97 & 0.55 \\
\hline Repeticiones & 2 & 1.55 & 12.05 & 0.84 \\
\hline Error & 14 & & & \\
\hline \multicolumn{2}{|c|}{ Coeficiente de Variación (\%): } & 18.80 & 9.64 & 19.29 \\
\hline
\end{tabular}

Tabla 10. Análisis de varianza para el rendimiento de hoja seca (Stevia rebaudiana), durante tres cortes productivos.

El desarrollo productivo de los tipos de esquejes que se evaluaron en esta investigación se observa en la Figura 12. Los esquejes conjuntamente con el tratamiento Testigo, después del corte de igualación presentan una proyección exponencial en el rendimiento de hoja seca en la primera cosecha. A partir de la segunda cosecha la producción se mantiene en cifras similares. La producción de la hoja de Stevia, obtenido por medio de la propagación de plantas de esqueje sub apical, presento los valores más altos en promedios de $810.99 \mathrm{KgHsha-1}$ en la primera cosecha, con una leve incremento productivo en la segunda cosecha de 839.19 KgHsha-1 y un rendimiento más alto en la tercera cosecha de 845.33 KgHsha-1. El testigo presento una proyección lineal en su producción de hoja seca con valores de 729.07 KgHsha-1 y 781.92 KgHsha-1 en el primer y segundo corte productivo y un rendimiento final en el tercer corte de

117 
828.82 KgHsha-1. De acuerdo a los resultados se confirma con Maya, 2003, quien menciona que los mejores rendimientos se obtienen a través de propagación vegetativa manifestando que las propiedades físicas priman sobre las químicas en los esquejes de Stevia reabudiana.

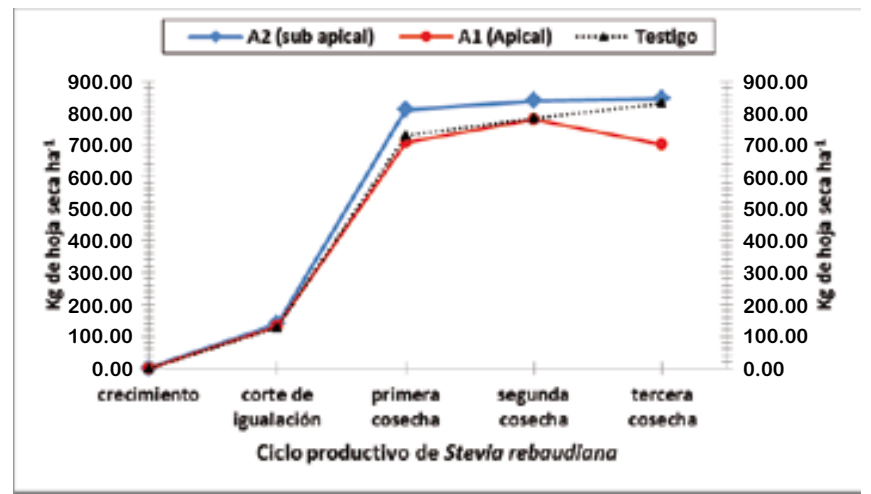

Figura 12. Rendimiento de hoja seca en dos tipos de esquejes de Stevia rebaudiana, durante el ciclo productivo.

La influencia de las diferentes dosis de potasio en el rendimiento de hoja seca de Stevia reabudiana fue imperceptible. En la Figura 13 se observan el comportamiento productivo de la Stevia a diferentes dosis de potasio, reportando un rendimiento parejo en comparación con el tratamiento Testigo. La dosis media de potasio (140 Kgha-1) arroja el mejor promedio en producción de hoja seca en la primera cosecha con $751.93 \mathrm{KgHsha-1}$ que difiere de las dosis baja (120 Kg ha-1) y alta (160 Kg ha-1). En la segunda cosecha la media es de 833.29 KgHsha-1 y en la última cosecha presento un promedio de 810.79 KgHsha-1. El testigo mantuvo una proyección lineal en su rendimiento con promedio de 729.07, 781.92 y 828.82 KgHsha-1 respectivamente.

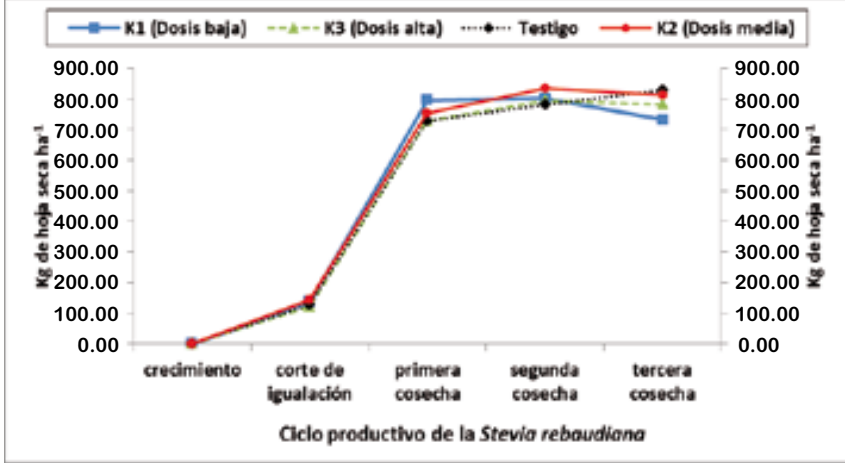

Figura 13. Influencia del potasio en el rendimiento de hoja seca de Stevia rebaudiana, durante el ciclo productivo.

El potasio tiene un importante efecto en la calidad de los órganos vegetativos. El uso de potasio es bien conocido en el incremento de tales factores como tamaño de flores, frutos, coloración y mantenimiento de la planta. El incremento de la calidad generalmente se lo consigue dentro del mismo rango de requerimiento para alcanzar los óptimos rendimientos (Clavijo, 1994).

A lo largo del ciclo productivo de la Stevia rebaudiana, se presenta valores medios en el rendimiento de hoja seca, la incidencia del potasio no es relevante en comparación con el testigo control. En la Figura 14 representamos el comportamiento productivo de la dosis media de potasio (140 Kgha-1) que mostro los mejores promedios en rendimiento de hoja seca y fresca. Como se puede observar existe un crecimiento lineal en biomasa de hoja fresca desde la primera cosecha hasta la segunda cosecha con valores de 2989 KgHfha-1 y 3933.87 KgHfha-1 respectivamente. En la tercera cosecha presenta un decrecimiento productivo con un promedio de $3182.77 \mathrm{KgHfha-1}$ similares al tratamiento testigo en materia fresca con 3123,59 KgHfha-1. Esto confirma lo citado por Taiz y Zeiger, 2006 quienes señalan que la hoja sería el órgano que menores niveles de migración presentaría dada la alta actividad fotosintética en pre-floración, donde es requerida una alta eficiencia de K en la apertura y cierre estomático para mantener el estado hídrico de la planta y para la activación de muchas enzimas esenciales para la fotosíntesis y la respiración. 
En la Figura 15 se observa que las plantas de Stevia provenientes del enraizamiento con esquejes de tipo sub apical, presenta el mejor rendimiento en hoja seca a diferencia de las provenientes de esqueje tipo apical. La producción de hoja fresca presenta su mayor promedio en la segunda cosecha con 3908.37 KgHfha-1. La primera y tercera cosecha se reportan valores similares de $3288.60 \mathrm{Kg}$ Hfha-1 y 3248.42 KgHfha-1. De acuerdo a lo mencionado, Ramesh et al, 2006, indica que en concordancia con la mayoría de las especies vegetales, el comportamiento de Stevia es afectado por la radiación, longitud del día, temperatura, agua disponible en el suelo y el viento en lugares expuestos. Se concluyen que la Stevia es una planta amante al sol, probablemente debido a que evolucionó en un ambiente cálido, húmedo y soleado.

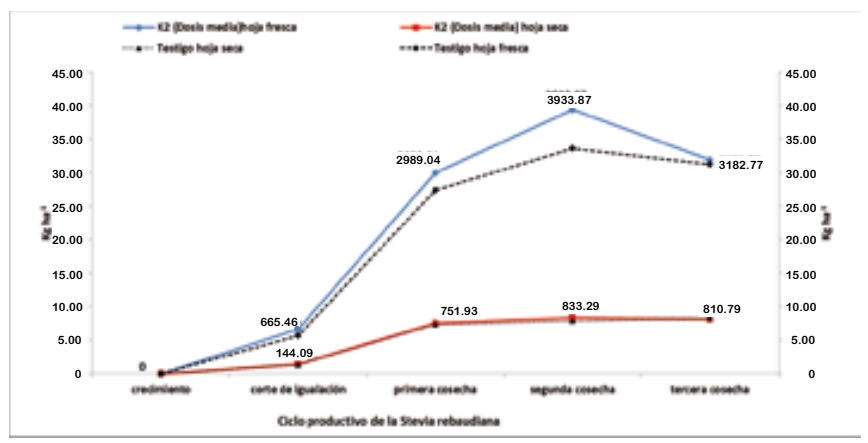

Figura 14. Influencia del potasio en el rendimiento de hoja fresca y seca de Stevia rebaudiana, durante el ciclo productivo.

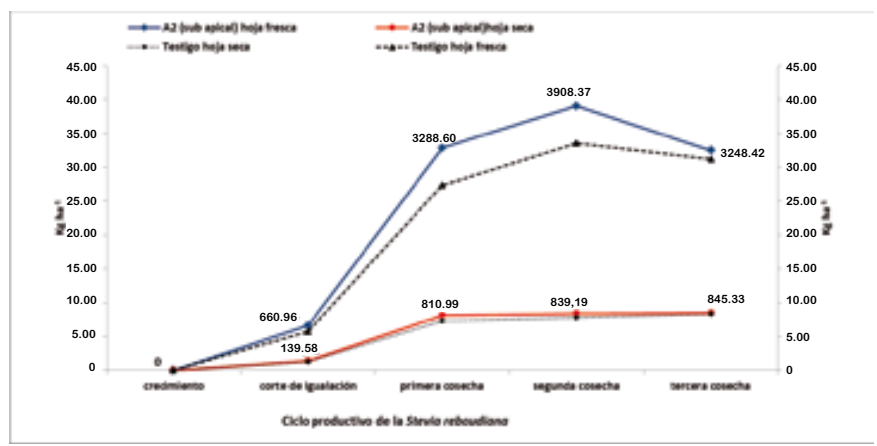

Figura 15. Producción del esqueje sub apical en hoja fresca y seca de Stevia rebaudiana, durante el ciclo productivo.

\section{MONITOREO DE PLAGAS Y ENFERMEDADES}

\section{Plagas}

Para esta variable se realizó observaciones semanales, durante la ejecución de la investigación, se suspendió el monitoreo al inicio de la cosecha, continuando al inicio del siguiente ciclo de producción. La incidencia de plagas en el cultivo de Stevia rebaudiana, fue esporádica, se observó la presencia de pequeños focos en el ensayo, sin presentar daños considerables en la planta, debido a su capacidad de recuperación vegetativa, manteniéndose bajo el umbral económico. En la Tabla 11, se reportan los resultados del monitoreo y la magnitud del ataque de los insectos plaga durante el desarrollo de esta investigación.

\begin{tabular}{|c|c|c|c|c|c|c|c|c|c|}
\hline \multirow{3}{*}{ Tratamientos } & \multicolumn{3}{|c|}{ Primera cosecha } & \multicolumn{3}{|c|}{ Segunda cosecha } & \multicolumn{3}{|c|}{ Tercera cosecha } \\
\hline & \multicolumn{3}{|c|}{$\%$} & \multicolumn{3}{|c|}{$\%$} & \multicolumn{3}{|c|}{$\%$} \\
\hline & Bajo & Medio & Alto & Bajo & Medio & Alto & Bajo & Medio & Alto \\
\hline T1 & 97.50 & 2.50 & 0.00 & 94.17 & 5.83 & 0.00 & 96.67 & 3.33 & 0.00 \\
\hline $\mathrm{T} 2$ & 97.50 & 2.50 & 0.00 & 90.83 & 9.17 & 0.00 & 95.83 & 4.17 & 0.00 \\
\hline T3 & 95.00 & 5.83 & 0.00 & 94.17 & 5.83 & 0.00 & 97.50 & 2.50 & 0.00 \\
\hline T4 & 92.50 & 7.50 & 0.00 & 93.33 & 6.67 & 0.00 & 95.83 & 4.17 & 0.00 \\
\hline T5 & 96.67 & 3.33 & 0.00 & 88.33 & 11.67 & 0.00 & 96.67 & 3.33 & 0.00 \\
\hline T6 & 96.67 & 4.17 & 0.00 & 90.83 & 8.33 & 0.00 & 96.67 & 3.33 & 0.00 \\
\hline T7 & 97.50 & 2.50 & 0.00 & 84.17 & 15.00 & 0.00 & 95.83 & 4.17 & 0.00 \\
\hline T8 & 98.33 & 1.67 & 0.00 & 81.67 & 18.33 & 0.00 & 95.83 & 4.17 & 0.00 \\
\hline
\end{tabular}

Tabla 11. Incidencia de ataque de plagas durante el ciclo de productivo de

Stevia rebaudiana

Según los resultados obtenidos se observa que la incidencia de insectos fue baja y consecutivamente desaparecieron en forma natural, debido a la condición fisiológica de recuperación. En las primeras etapas fenológicas del cultivo la presencia de plagas es notable, en especial de grillo topo gryllotalpa sp, luego desaparece conforme la planta se vuelve longeva. Las plagas de tipo chupadoras y raspadoras causan daño indirecto, pues se alimentan de la savia o del jugo celular, disminuyendo el crecimiento de la planta por reducción de foto asimilados disponibles para los procesos metabólicos. Además en el cultivo observamos la presencia de hormigas arriera (Atta sp) que no causo daños en la hoja a lo largo del cultivo. 
Influencia de tres fitohormonas para la propagación vegetativa sobre dos tipos de esquejes de Ka' a he' e (Stevia rebaudiana), con tres dosis de potasio y su efecto sobre el rendimiento del cultivo en la zona de Santo Domingo de los Tsáchilas.

\section{Enfermedades}

La presencia de enfermedades de acuerdo al monitoreo y seguimiento, durante el ciclo productivo de la Stevia rebaudiana, fue leve, provocado en parte a los cambios drásticos del clima y a la arquitectura fisiológica de la planta, factores que intervinieron en el control de las mismas. En la Tabla 12, se visualiza la incidencia de las enfermedades presentes en el cultivo. El control sanitario se hizo evidente como un medio preventivo que favoreció el desarrollo optimo del cultivo en combinación con labores culturales que evitaron el uso innecesario de productos químicos.

\begin{tabular}{|c|c|c|c|c|c|c|c|c|c|}
\hline & \multicolumn{3}{|c|}{ Primera cosecha } & \multicolumn{3}{|c|}{ Segunda cosecha } & \multicolumn{3}{|c|}{ Tercera cosecha } \\
\hline \multirow{2}{*}{ Tratamientos } & \multicolumn{3}{|c|}{$\%$} & \multicolumn{3}{|c|}{$\%$} & \multicolumn{3}{|c|}{$\%$} \\
\hline & Bajo & Medio & Alto & Bajo & Medio & Alto & Bajo & Medio & Alto \\
\hline T1 & 59.17 & 38.33 & 3.33 & 64.17 & 31.67 & 4.17 & 60.00 & 40.00 & 0.00 \\
\hline $\mathrm{T2}$ & 53.33 & 43.33 & 3.33 & 53.33 & 42.50 & 4.17 & 50.83 & 49.17 & 0.00 \\
\hline T3 & 65.83 & 30.83 & 3.33 & 71.67 & 23.33 & 5.83 & 65.00 & 35.00 & 0.00 \\
\hline T4 & 62.50 & 35.83 & 1.67 & 70.00 & 26.67 & 3.33 & 60.00 & 40.00 & 0.00 \\
\hline T5 & 67.50 & 37.50 & 2.50 & 68.33 & 28.33 & 3.33 & 71.67 & 26.67 & 1.67 \\
\hline T6 & 74.17 & 24.17 & 1.67 & 76.67 & 19.17 & 4.17 & 76.67 & 23.33 & 0.00 \\
\hline T7 & 52.50 & 44.17 & 3.33 & 54.17 & 38.33 & 7.50 & 52.50 & 47.50 & 0.00 \\
\hline T8 & 59.17 & 39.17 & 1.67 & 63.33 & 30.00 & 6.67 & 57.50 & 42.50 & 0.00 \\
\hline
\end{tabular}

Tabla 12. Incidencia de enfermedades, durante el ciclo de productivo de Stevia reabudiana

En la primera y segunda cosecha, presentaron casos esporádicos donde la planta se encontró afectada en más del $50 \%$ de sus órganos vegetativos, en tanto que en la tercera cosecha la incidencia de enfermedades es nula. Las enfermedades que presentaron durante el ciclo productivo de la Stevia fueron: Seda blanca Sclerotium rolfsi, y la pudrición violácea (Alternaria sp, Rhizoctonia sp); presentes en forma dispersa. En la etapa de crecimiento de la planta hubo presencia de Oidium sp, la que se extingue conforme la planta alcanza la madurez fisiológica, concordando con Albán, 2010, quien manifiesta que las enfermedades relevantes de la Stevia son: Mancha foliar, ocasionada por Septoria sp; Cenicilla, ocasionada por Oidium sp; Pudrición causada por Sclerotium rolfsi, y Rhizoctonia sp.

\section{CONCENTRACION DE POTASIO (K)}

\section{Porcentaje de Potasio en la hoja de Stevia reabudiana}

En la Tabla 13, se observa la concentración de K en las hojas de Stevia, en los diferentes ciclos productivos y durante el desarrollo de la investigación. Las hojas fueron el órgano, que menor nivel de migración presento, dada la alta actividad fotosintética en prefloración, donde es requerida una alta eficiencia de K en la apertura estomática y en la activación de un gran número de enzimas. El tratamiento T6 presento las mayores concentraciones de K en la hoja (materia seca), con $4.3 \%$ de $\mathrm{K}$ en la primera cosecha, $6.8 \%$ de $\mathrm{K}$ en la segunda cosecha y $5.8 \%$ de $\mathrm{K}$ en la ultima cosecha. El K aumenta el área de la hoja y el contenido de clorofila, retrasa su senectud y por eso contribuye a un crecimiento de la capa superior de sus hojas por la fotosíntesis del cultivo.

\begin{tabular}{l|c|c|c|c}
\hline \multicolumn{2}{c}{ Tratamientos } & \multicolumn{3}{c}{ Concentración de K (\% de materia seca) } \\
\hline Esqueje & $\begin{array}{l}\text { Dosis K } \\
\left.\text { Kgha }^{-1}\right)\end{array}$ & Primera cosecha & Segunda cosecha & Tercera cosecha \\
\hline Apical & 120 & 4.4 & 5.7 & 4.5 \\
Apical & 140 & 4.5 & 4.0 & 5.8 \\
Apical & 160 & 2.8 & 4.3 & 5.9 \\
Sub apical & 120 & 3.0 & 3.0 & 4.6 \\
Sub apical & 140 & 4.2 & 4.8 & 5.1 \\
Sub apical & 160 & 4.3 & 6.8 & 5.8 \\
Testigo 1 & & 2.6 & 3.4 & 4.0 \\
Testigo 2 & & 2.6 & 3.0 & 4.3 \\
\hline
\end{tabular}

Tabla 13. Concentración de K (\% de materia seca) en la hoja de Stevia rebaudiana

En la Figura 16 se observa las concentraciones de K (potasio) en la hojas de Stevia rebaudiana de acuerdo con las diferentes Dosis de $\mathrm{K}$ que evaluaron en el ensayo. El tratamiento Testigo mantienen una concentración baja de $\mathrm{K}$ con tendencia lineal conforme el cultivo se vuelve longevo y su respuesta fisiológica demanda mayor concentración de este elemento de 2,6 \% de K en materia seca en la primera cosecha, $3.2 \%$ de $\mathrm{K}$ en la segunda cosecha y en la tercera cosecha se presento la mayor concentración promedio de $4.2 \%$ de $\mathrm{K}$ en materia seca de la hoja. Las dosis de $\mathrm{K}$ reportaron mayores promedios de concentración en la hoja durante el ciclo productivo

120 
de la Stevia, la dosis de potasio media (140 Kgha-1) en la primera cosecha, se mantuvo con la mayor concentración que fue de 4.4 \% de $\mathrm{K}$ en promedio. En la segunda y tercera cosecha de hoja de stevia el análisis reporto los mayores niveles con la dosis alta de potasio (160 Kgha-1) con concentraciones de 5.6 y $5.9 \%$ de K en materia seca.

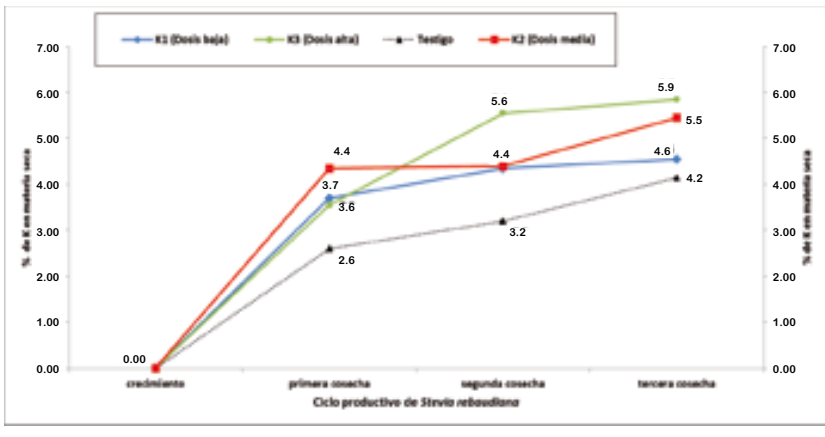

Figura 16. Concentración de K (\% de materia seca) en la hoja de Stevia rebaudiana a diferentes dosis de Potasio.

\section{Factor parcial de productividad (K)}

En la Tabla 14 se muestra, la relación de Kg de hoja seca cosechada por $\mathrm{Kg}$ de $\mathrm{K}$ aplicado evaluados en los diferentes tratamientos. El tratamiento T4 (esqueje sub apical - $120 \mathrm{Kg}$ de Kha-1) reporta el mejor factor de productividad durante el ciclo productivo de la Stevia rebaudiana; los valores promedios obtenidos en este ensayo son: 7.31, 7.07 y $6.88 \mathrm{KgHs} / \mathrm{KgK}$ aplicado en la primera, segunda y tercera cosecha respectivamente.

\begin{tabular}{lcccc}
\hline \multicolumn{2}{l}{$\begin{array}{l}\text { Tratamientos } \\
\text { Esqueje }\end{array}$} & \multicolumn{3}{c}{$\begin{array}{c}\text { Factor parcial de productividad } \\
(\mathrm{Kg} \text { cosechado/Kg el emento aplicado) }\end{array}$} \\
\hline Apical & 120 & 5.96 & 6.26 & 5.30 \\
Apical & 140 & 5.23 & 5.59 & 5.17 \\
Apical & 160 & 4.19 & 5.01 & 4.66 \\
Sub apical & 120 & 7.31 & 7.07 & 6.88 \\
Sub apical & 140 & 5.51 & 6.31 & 6.41 \\
Sub apical & 160 & 4.90 & 4.91 & 5.08 \\
Testigo 1 & & 5.83 & 6.32 & 7.43 \\
Testigo 2 & & 5.29 & 5.63 & 5.38 \\
\hline
\end{tabular}

Tabla 14. Factor parcial de productividad (K) en el ciclo productivo de la Stevia
El exceso de potasio en principio no ocasiona ningún daño, el efecto perjudicial podría producirse por el efecto antagónico que tiene con el magnesio, dificultando la absorción de este nutriente. El potasio es muy rápidamente absorbido y la mayor parte está en el componente líquido de la célula en forma soluble. Es un elemento muy móvil en la planta y se mueve sueltamente desde los tejidos viejos hacia los puntos de crecimiento de raíces y parte aérea.

\section{Eficiencia Agronómica}

La eficiencia agronómica revela la relación entre el aumento en la producción (Kg) por la cantidad de elemento aplicado (Kg), de acuerdo a la Tabla 15, los tratamientos con dosis baja de potasio (120 KgKha-1)muestran los valores óptimos durante el ciclo agroproductivo de la Stevia rebaudiana, el T1 (esqueje apical - 120 KgKha-1), para la primera, segunda y tercera cosecha presentaron promedios de 10.35, 10.87 y $9.21 \mathrm{Kg}$ de aumento en producción/KgK aplicado, en tanto que el T4 (esqueje sub apical - 120 KgKha-1), muestran valores de 14.49, 14.01 y $13.63 \mathrm{Kg}$ de aumento en producción/KgK aplicado, en las tres cosechas respectivamente. El potasio es un regulador fisiológico en las plantas y su movilidad entre los tejidos vegetales, permite actuar sobre la turgencia celular. Es importante además en la producción, eficiencia de uso y en el desplazamiento de los asimilados a los órganos de almacenamiento.

\begin{tabular}{|c|c|c|c|c|}
\hline \multicolumn{2}{|c|}{ Tratamientos } & \multicolumn{3}{|c|}{$\begin{array}{c}\text { Eficiencia agronómica } \\
\text { (Kg aumento en la producción } / \mathrm{Kg} \text { elemento aplicado) }\end{array}$} \\
\hline Esqueje & Dosis $\mathrm{K}\left(\mathrm{Kg} \mathrm{ha}^{-1}\right)$ & Primera cosecha & Segunda cosecha & Tercera cosecha \\
\hline Apical & 120 & 10.35 & 10.87 & 9.21 \\
\hline Apical & 140 & 5.39 & 5.76 & 5.32 \\
\hline Apical & 160 & 7.28 & 8.69 & 8.10 \\
\hline Sub apical & 120 & 14.49 & 14.01 & 13.63 \\
\hline Sub apical & 140 & 6.03 & 6.90 & 7.02 \\
\hline Sub apical & 160 & 10.27 & 10.29 & 10.63 \\
\hline Testigo 1 & & 5.87 & 6.36 & 7.47 \\
\hline Testigo 2 & & 5.55 & 5.90 & 5.65 \\
\hline
\end{tabular}

Tabla 15. Eficiencia agronómica de potasio (K) en el ciclo productivo de la Stevia rebaudiana.

rebaudiana. 
Influencia de tres fitohormonas para la propagación vegetativa sobre dos tipos de esquejes de Ka' a he' e (Stevia rebaudiana), con tres dosis de potasio y su efecto sobre el rendimiento del cultivo en la zona de Santo Domingo de los Tsáchilas.

El potasio, es en muchas ocasiones, tomado más tempranamente que el nitrógeno y el fósforo y su asimilación se incrementa más rápido que la producción de materia seca. Esto significa que el potasio se acumula temprano en el período de crecimiento y luego es traslocado a otras áreas

\section{Eficiencia fisiológica}

En la Tabla 16, reporta la eficiencia fisiológica de la Stevia rebaudiana, es decir la relación a la respuesta de cuantos kilogramos de hoja seca que es el órgano de interés económico de este cultivo se producen a partir de la absorción de K en la planta. Los tratamientos testigos mantiene la mejor eficiencia fisiológica en absorción de nutrientes, necesarios en el ciclo agroproductivo de la planta, con valores de $38.46 \mathrm{KgHs} / \mathrm{KgK}$ absorbido en la primera cosecha, en tanto que la segunda cosecha presentan promedios de 29.41 y $33.33 \mathrm{KgHs} /$ KgK absorbido. En la última cosecha se observa reducción en su eficiencia si se compara con las anteriores de 25.00 y $23.26 \mathrm{KgHs} / \mathrm{KgK}$ absorbido.

\begin{tabular}{lcccc}
\hline \multirow{2}{*}{ Tratamientos } & \multicolumn{3}{c}{$\begin{array}{c}\text { Eficiencia fisiológica } \\
(\text { Kg de producción/Kg de K absorción) }\end{array}$} \\
\hline Esqueje & Dosis K $\left(\mathrm{Kg} \mathrm{ha}^{-1}\right)$ & Primera cosecha & Segunda cosecha & Tercera cosecha \\
\hline Apical & 120 & 22.73 & 17.54 & 22.22 \\
Apical & 140 & 22.22 & 25.00 & 17.24 \\
Apical & 160 & 35.71 & 23.26 & 16.95 \\
Sub apical & 120 & 33.33 & 33.33 & 21.74 \\
Sub apical & 140 & 23.81 & 20.83 & 19.61 \\
Sub apical & 160 & 23.26 & 14.71 & 17.24 \\
Testigo 1 & & 38.46 & 29.41 & 25.00 \\
Testigo 2 & & 38.46 & 33.33 & 23.26 \\
\hline
\end{tabular}

Tabla 16. Eficiencia fisiológica (K) en el ciclo productivo de la Stevia rebaudiana.

El potasio controla muchas funciones fisiológicas, como la turgencia de las plantas, formación de materia grasa, proteínas, clorofila, carbohidratos, respiración y regulación de la transpiración del agua, entre otras.

\section{Eficiencia aparente de recuperación}

No se observa diferencias significativas entre los tratamientos de acuerdo a la Tabla 17, en cuanto a la recuperación de K aplicado y la absorción de la planta, con el fin de generar biomasa. Los tratamientos Testigo ( $\left.\begin{array}{lll}1 & \text { y } & 2\end{array}\right)$ mantienen la eficiencia más baja en comparación con el resto de tratamientos en la primera y segunda cosecha con concentraciones de 0.15 a 0.14 Kg aumento en absorción/ $\mathrm{Kg} \mathrm{K}$ aplicado en el primer corte y en el segundo corte se reporto entre 0.21 y $0.17 \mathrm{Kg}$ aumento en absorción/Kg K aplicado. En la tercera cosecha los niveles no difieren del resto con valores de 0.30 y 0.23 en eficiencia recuperación de la Stevia rebaudiana. El potasio ayuda en la asimilación de la luz aumentando el proceso fotosintético, y siendo menos necesario cuando el período luminoso es alto o normal. La Stevia es una especie vegetal de foto-período corto y su producción depende de la cantidad de luz día que reciba.

\begin{tabular}{|c|c|c|c|c|}
\hline \multicolumn{2}{|c|}{ Tratamientos } & \multicolumn{3}{|c|}{$\begin{array}{c}\text { Eficiencia aparente de recuperación } \\
\text { (Kg aumento en absorción/Kg K aplicado) }\end{array}$} \\
\hline Esqueje & Dosis $\mathrm{K}\left(\mathrm{Kg} \mathrm{ha}^{-1}\right)$ & Primera cosecha & Segunda cosecha & Tercera cosecha \\
\hline Apical & 120 & 0.26 & 0.36 & 0.24 \\
\hline Apical & 140 & 0.24 & 0.22 & 0.30 \\
\hline Apical & 160 & 0.12 & 0.22 & 0.28 \\
\hline Sub apical & 120 & 0.22 & 0.21 & 0.32 \\
\hline Sub apical & 140 & 0.23 & 0.30 & 0.33 \\
\hline Sub apical & 160 & 0.21 & 0.33 & 0.29 \\
\hline Testigo 1 & & 0.15 & 0.21 & 0.30 \\
\hline Testigo 2 & & 0.14 & 0.17 & 0.23 \\
\hline
\end{tabular}

Tabla 17. Eficiencia aparente de recuperación (K) en el ciclo productivo de la Stevia rebaudiana.

El potasio está directamente asociado con el fortalecimiento de tejidos y es necesario para el desarrollo normal de lignina y celulosa, polisacáridos que dan fortaleza y rigidez a las plantas para mantenerse erguidas. El potasio incentiva el desarrollo del sistema radicular, lo cual da como resultado una mejor exploración de nutrientes y de agua en el suelo. El potasio tiene un importante efecto en la calidad de las hojas generalmente se lo consigue dentro del mismo rango de requerimiento para alcanzar los óptimos rendimientos. 


\section{Conclusiones}

De acuerdo con los resultados se concluye en la etapa de vivero:

El mayor porcentaje de enraizamiento se obtuvo con Raizal (A3) con $99.42 \%$ y Hormonagro (A1) con $97.17 \%$ en promedios. Valores que favorecen el enraizamiento gracias a la acción de las diferentes concentraciones de auxinas sintéticas con el ácido naftalenacético (ANA) o ácidos orgánicos y en menor grado el ácido indolacético (AIA).

Los esquejes de tipo apical reportan las mejores condiciones de rizogénesis con un promedio de $\mathbf{9 7 . 7 2 \% ~ e n ~ p r e n d i m i e n t o ~ a ~ l o s ~} 15$ días de evaluación de Stevia rebaudiana.

El Factor A1 (Hormonagro) mantuvo el mayor número de raíces por esqueje de Stevia rebaudiana, que se evaluó a los 21 días con un promedio de 23.47 raíces/esqueje.

El menor tiempo de trasplante de esquejes (factor B) al campo definitivo fue de 23.05 días en promedio que corresponde al Factor A1 (Hormonagro).

En el campo definitivo se concluye que:

No se observaron diferencias significativas en la influencia de la dosis de potasio (K) y los diferentes tipos de esquejes en la plantación definitiva; en las altura de la plantas de Stevia rebaudiana, evaluados en los tres cortes productivos durante el desarrollo de la investigación.

El tipo de esqueje sub apical presento las mejores condiciones en el desarrollo de diámetro de tallo con promedios de $0.51 \mathrm{~cm}$ en la primera cosecha y $0.83 \mathrm{~cm}$ en la segunda cosecha.
La dosis baja y media de potasio presentaron influencia en la recuperación vegetativa de la Stevia rebaudiana durante la primera cosecha, con un promedios de 9.46 brotes/planta y 9.28 brotes/ planta, mientras que en la segunda y tercera cosecha la influencia de las dosis de potasio no fue significativa.

Los esquejes sub apicales presentaron las mejores condiciones de proliferación en el ensayo, con una recuperación vegetativa de: 9,60 brotes/planta, 33.91 brotes/planta y 55.12 brotes/planta.

La dosis de potasio evaluados en el rendimiento productivo, no reporto significancia estadística en acumulación y formación de materia seca.

La Stevia rebaudiana es una planta ruderal que se comporta como una maleza y por ello en su estado natural, la especie crece en suelos ácidos de baja fertilidad de tipo arenoso y con alta humedad. Esta amplia gama de supervivencia le permite adaptarse a medios poco favorables.

La Stevia es una especie vegetal de foto-período corto y su producción depende de la cantidad de luz día que reciba y es probable que condiciones distintas al foto periodo (radiación solar) y humedad limiten la producción en la zona.

No hay interacciones presentes en este ensayo entre los tipos de esquejes y las diferentes dosis de potasio que se probaron en las variables altura de planta, diámetro de tallo, recuperación vegetativa y rendimiento.

La incidencia de plagas y enfermedades fue intermitente en todo el año de evaluación de la planta, bajo las condiciones ambientales de la zona. El ciclo de cultivo predispone en los primeros estadios para el ataque de plagas mientras que en las enfermedades la calidad fenotípica de la planta y los factores ambientales (humedad, temperatura etc.) determina la severidad del ataque.

123

\section{Tsafiqui凿}


Influencia de tres fitohormonas para la propagación vegetativa sobre dos tipos de esquejes de Ka' a he' e (Stevia rebaudiana), con tres dosis de potasio y su efecto sobre el rendimiento del cultivo en la zona de Santo Domingo de los Tsáchilas.

\section{Bibliografía}

Alban, A, 2010, Tesis de grado: Evaluación de 4 dosis de humus, en la adaptación del cultivo no tradicional de Ka' a he' e (Stevia rebaudiana) Yerba dulce en la zona de Santo Domingo de los Colorados, Universidad Tecnológica Equinoccial, Santo Domingo - Ecuador, pág.45.

Ballevé, R.M.L., 1992. Marcadores Isoenzimáticos y Biología de Reproducción de Stevia rebaudiana. Tese de Doutl. UNICAMP-IB.

Birchler t, 1998. La planta ideal: Revisión del concepto, parámetros definitorios e implementación práctica. Nursery Technology Cooperative. Oregon State University Forest Sciences Lab 020. Corvallis. Oregon 97331 - 7501. EE.UU.

Bañón, S., Martínez-Sánchez, J.J., Fernández, J.A., González, A. and OCHOA, J. 2002.Effect of indolebutyric acid and paclobutrazol on the rooting of Rhamnus alaternus stem cuttings. Acta Hortic. 614: 263-267.

Bredmose, N., Hansen, J., Nielsen, J., Zieslin, N. 2001. Topophysic influences on rose bud and shoot growth and flower development are determined by endogenous axillary bud factors. Acta-Horticulturae $n^{\circ}$ 547: 177-183.

Cabello, A. 2000. Propagación Asexual. Apuntes de Clases $N^{\circ} 2$. Departamento de Silvicultura. Facultad de Ciencias Forestales. Universidad de Chile. $10 \mathrm{p}$.

Clavijo Jairo, 1994. Metabolismo de los Nutrientes en las Plantas en Fertilidad de Suelos. Diagnóstico y Control. Sociedad Colombina de la Ciencia del Suelo. Bogotá. Colombia, pp. 14 - 28.

Enríquez, J. 2003, Experiencias de propagación in vitro de plantas, Instituto Tecnológico Agropecuario de Oaxaca, Serie de Cuadernos de los Centros: 1- 37 p. KAFKAFI, U. and Xu, G.H. 1999. Potassium nutrition for high crop yields. In: rontiers in potassium nutrition: new perspectives on the effects of potassium on physiology of plants (D. M. Oosterhuis, and G. Berkowitz, eds.). 133-142: PPI/PPIC, Georgia, USA.

Katayama O., Sumida, T, Hayashi, H. and Mitsuhashi H. 1976. The practical application of Stevia and research and development data (English translation). I.S.U. Company, Japan. 747 pp.

Martínez, J.V.2000 Fundamentos de Agrotecnología de cultivo de plantas medicinales iberoamericanas. CYTED. Cooperación Iberoamericana. Convenio Andrés Bello.

Maya, D.L, 2003, Stevia rebaudiana, Bertoni. Kaá-he. Monografía. Publicación de la Secretaria de Agricultura de Antioquia. 21 p.

Ramesh, K., V. Singh y N. W. Megeji. 2006. Cultivation of stevia [Stevia rebaudiana (bert.) Bertoni]: A comprehensive review. En: Advances in Agronomy Vol. 89. 360 p. Academic Press. San Diego, California - USA.

Taíz, L. y E. Zeiger. 2006. Essensial nutrients, deficiencies and plant dissorders. En: Plant Physiology. IV ed. Sinauer Associates, Inc. p. 75-83.

Vivanco, 2009. Tesis de grado: Evaluación de la Eficacia del Bioplus, Hormonagro y Enraizador Universal en la propagación asexual de Hypericum (Hypericum ssp.), Riobamba - Ecuador, Escuela Superior Politécnica de Chimborazo, Pág. 38, 39, 40, 60. 


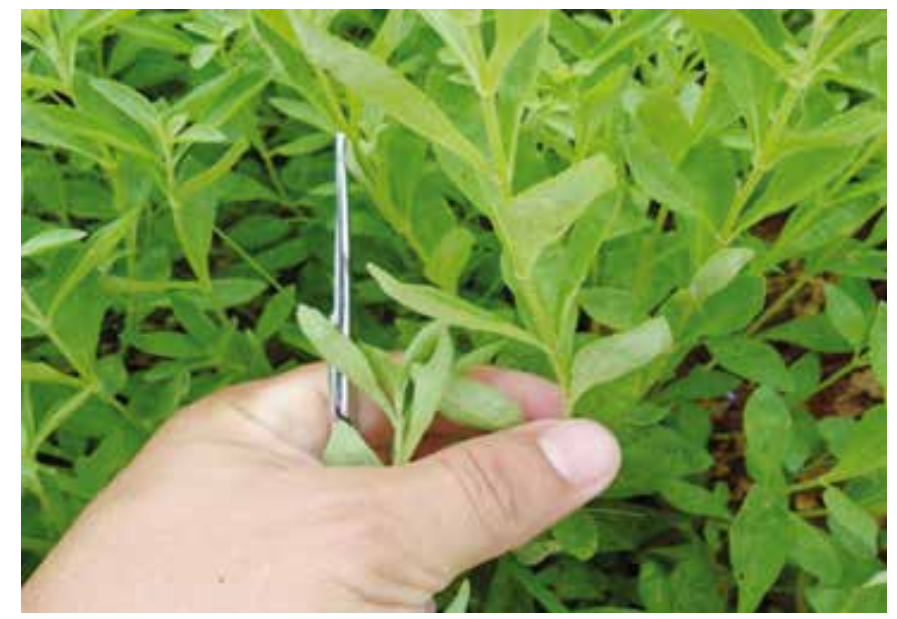

Foto $N^{\circ}$ 1. Plantas madres de Stevia

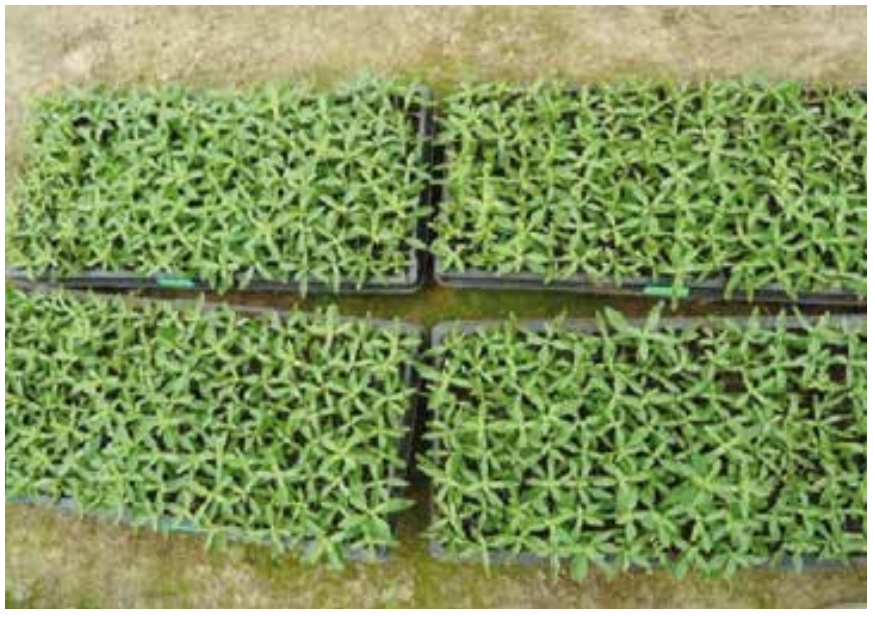

Foto $\mathrm{N}^{\circ} 3$. Disposición de tratamientos

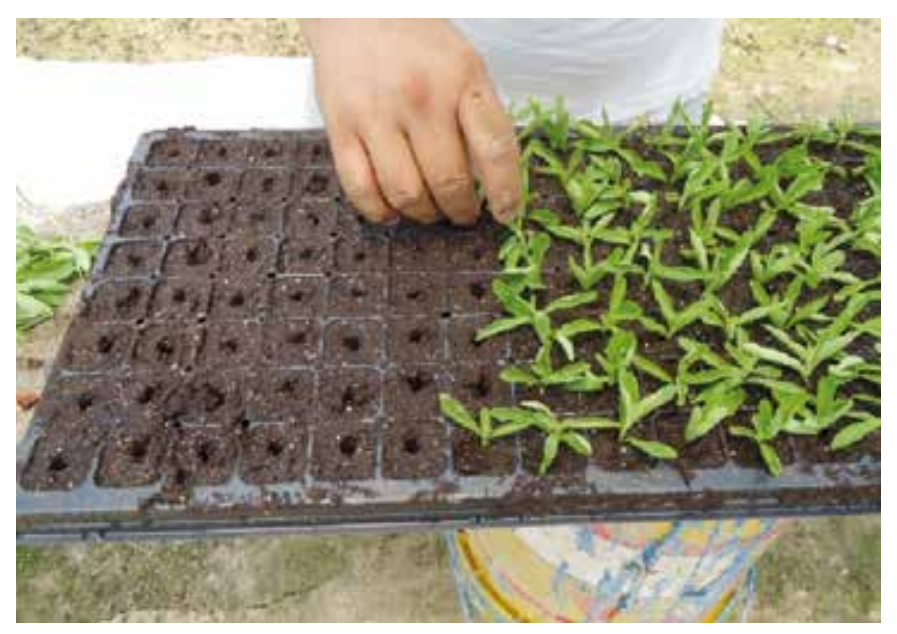

Foto $\mathrm{N}^{\circ} 2$. Siembra de esquejes

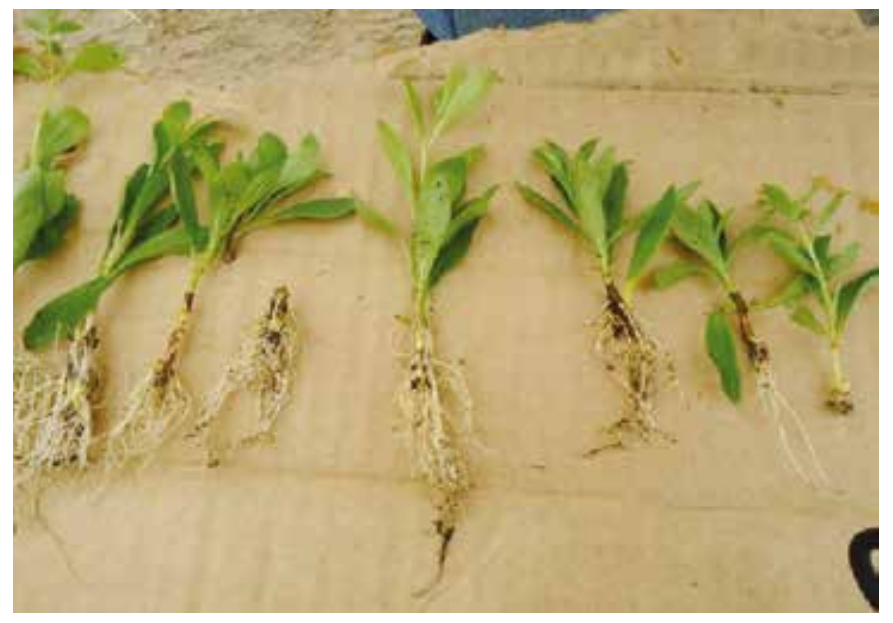

Foto $\mathrm{N}^{\circ} 4$. Enraizamiento de esquejes 
Influencia de tres fitohormonas para la propagación vegetativa sobre dos tipos de esquejes de Ka' a he' e (Stevia rebaudiana), con tres dosis de potasio y su efecto sobre el rendimiento del cultivo en la zona de Santo Domingo de los Tsáchilas.

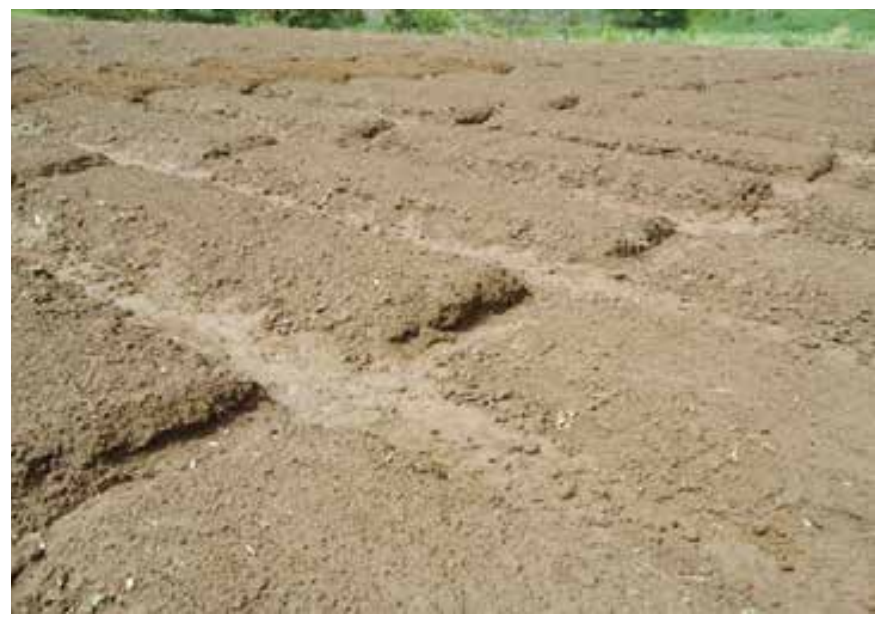

Foto $\mathrm{N}^{\circ} 5$. Disposición de los tratamientos

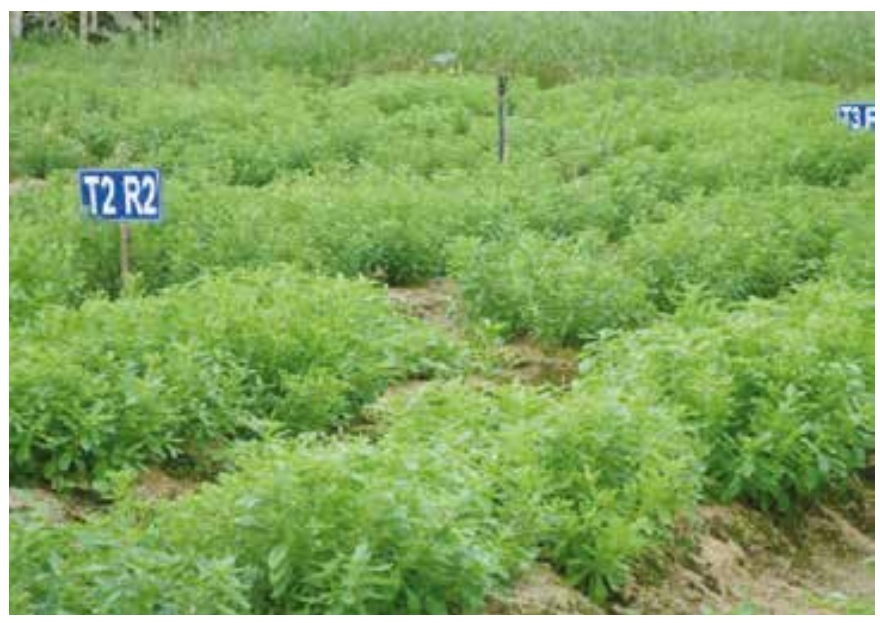

Foto $\mathrm{N}^{\circ} 7$. Desarrollo del cultivo de Stevia

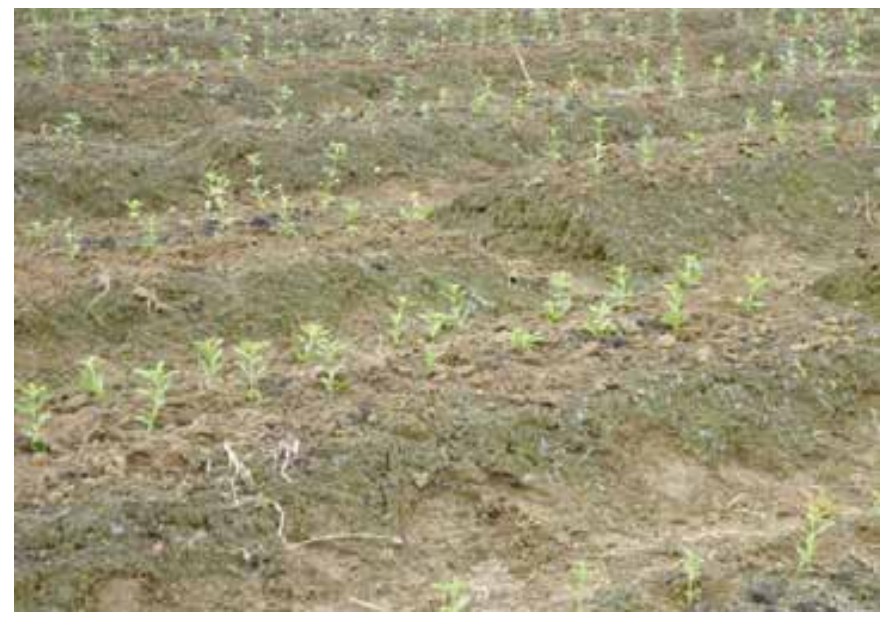

Foto $N^{\circ} 6$. Siembra de plantines de Stevia

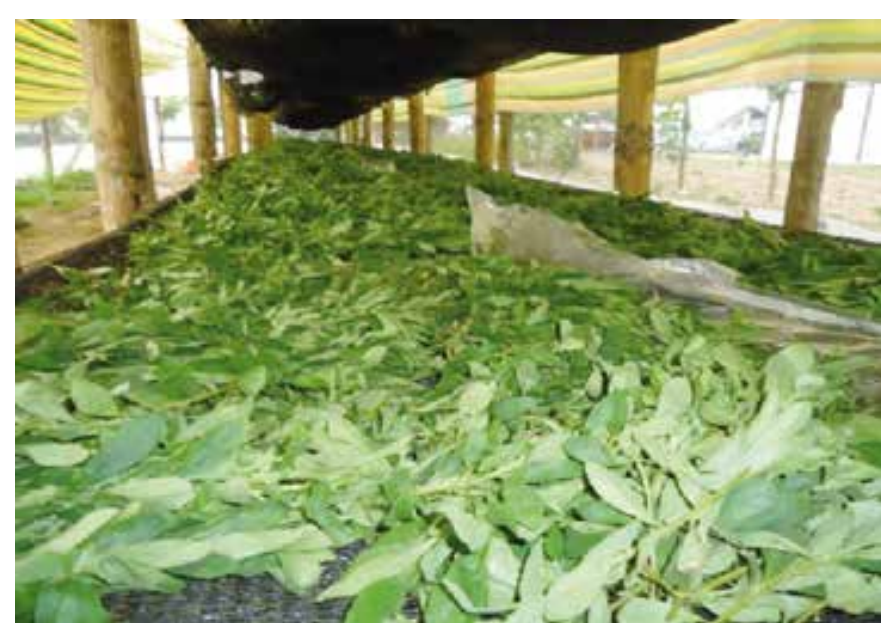

Foto $N^{\circ} 8$. Secado de la hoja de Stevia 